\title{
Luminescence as a Sediment Tracer and Provenance Tool
}

\section{Gray, Harrison J.; Jain, Mayank; Sawakuchi, Andre O.; Mahan, Shannon A.; Tucker, Gregory E.}

Published in:

Reviews of Geophysics

Link to article, DOI:

10.1029/2019RG000646

Publication date:

2019

Document Version

Publisher's PDF, also known as Version of record

Link back to DTU Orbit

Citation (APA):

Gray, H. J., Jain, M., Sawakuchi, A. O., Mahan, S. A., \& Tucker, G. E. (2019). Luminescence as a Sediment Tracer and Provenance Tool. Reviews of Geophysics, 57(3), 987-1017. https://doi.org/10.1029/2019RG000646

\section{General rights}

Copyright and moral rights for the publications made accessible in the public portal are retained by the authors and/or other copyright owners and it is a condition of accessing publications that users recognise and abide by the legal requirements associated with these rights.

- Users may download and print one copy of any publication from the public portal for the purpose of private study or research.

- You may not further distribute the material or use it for any profit-making activity or commercial gain

- You may freely distribute the URL identifying the publication in the public portal 


\section{Reviews of Geophysics}

\author{
REVIEW ARTICLE \\ 10.1029/2019RG000646 \\ Key Points: \\ - Luminescence, a property of matter \\ often used for dating, can also be \\ used as a sediment tracer and \\ sediment fingerprinting tool \\ - We review previous research on \\ luminescence sediment \\ tracing/fingerprinting and show that \\ there is a significant body of \\ research \\ - There is potential to expand and \\ develop the luminescence sediment \\ tracing/fingerprinting method to \\ various geomorphic environments
}

Correspondence to:

H. J. Gray,

harrison.gray@colorado.edu

Citation:

Gray, H. J., Jain, M., Sawakuchi, A. O. Mahan, S. A., \& Tucker, G. E. (2019). Luminescence as a sediment tracer and provenance tool. Reviews of Geophysics, 57. https://doi.org/10.1029/

2019RG000646

Received 23 FEB 2019

Accepted 12 JUL 2019

Accepted article online 30 JUL 2019

(C)2019. American Geophysical Union. All Rights Reserved.

\section{Luminescence as a Sediment Tracer and Provenance Tool}

\author{
Harrison J. Gray ${ }^{1}$ (D), Mayank Jain ${ }^{2}$, Andre O. Sawakuchi ${ }^{3}$ (D), Shannon A. Mahan' ${ }^{1}$, \\ and Gregory E. Tucker ${ }^{4}$ iD \\ ${ }^{1}$ Luminescence Geochronology Laboratory, Geosciences and Environmental Change Science Center, U.S. Geological \\ Survey, Denver, CO, USA, ${ }^{2}$ Center for Nuclear Technologies, Technical University of Denmark, DTU Risø Campus, \\ Roskilde, Denmark, ${ }^{3}$ Department of Sedimentary and Environmental Geology, Institute of Geosciences, University of São \\ Paulo, São Paulo, SP, Brazil, ${ }^{4}$ Cooperative Institute for Research in Environmental Sciences (CIRES) and Department of \\ Geosciences, University of Colorado Boulder, Boulder, CO, USA
}

\begin{abstract}
Luminescence holds unique potential as a sediment tracer and provenance method. The tracer application of luminescence has key advantages including ease of measurement, relatively low cost, and applicability to geologically ubiquitous quartz and feldspar sand and silt. These advantages can help answer fundamental questions about geomorphology, sediment transport, sediment production, and the tectonic/climatic controls on source-to-sink sedimentary systems. There is a notable body of research on luminescence as a sediment tracer. These tracer methods range from identifying source locations based on unique luminescence characteristics, to observing changes in luminescence characteristics with transport, to using residual luminescence to infer rates of transport. Previous applications of luminescence include provenance and quantification of fluvial transport rate, tracing of coastal longshore drift, estimations of mixing rates in soil or sediment, and provenance of wind-blown deposits. The few studies that compare luminescence methods with nonluminescence tracer methods show good agreement. However, more work is needed to test the application of luminescence tracers in sediments. Future research directions should focus on comparing luminescence-based with nonluminescence tracer methods. Furthermore, research is needed on the effects of specific geomorphic processes on luminescence characteristics and residual doses. While there is significant potential for future research, luminescence is already a useful sediment tracer and provenance tool applicable to a wide range of geomorphic environments.
\end{abstract}

Plain Language Summary We live on a surface that is constantly changing. These changes occur as sediment is moved around by various forces in the environment. We want to be better able to predict sediment movement so we can minimize its negative effects. Examples of these negative effects include erosion of soil on farms, filling of reservoirs we use for water with sediment, and pollution of sediment in waterways and ecosystems. To get better at predicting negative effects, we need to know how fast sediment moves and where it comes from. Some techniques scientists use to answer these questions include using unique properties of sand grains to track their movement. However, there is not a technique that works for every environment, so we have to develop new techniques to expand our capability. In this paper, we review the use of luminescence, a property that changes in sunlight, to track the movements of sediment.

Luminescence is not commonly used as a sediment tracker but shows lots of potential. We outline the past uses of luminescence as a sediment tracker and describe the future research that is needed to improve its use as a tool for scientists.

\section{Introduction}

The movement of sediment across the Earth is a fundamental process that lies at the interface between the biological and geological worlds. From a human perspective, sediment transport can deliver nutrients that nourish soil and feed society, yet also bury houses and destroy livelihoods (Burbank \& Anderson, 2009). From a geological perspective, the movement of sediment transforms landscapes as a response to climatic fluidity and tectonic unrest (Anderson \& Anderson, 2010). These perspectives together encompass a variety of problems both practical, such as soil erosion (Guzmán et al., 2013), reservoir sedimentation (Syvitski et al., 2005), floods, contaminant/nutrient dispersion (Pizzuto et al., 2014), desertification; and fundamental, including modeling landscape evolution (Tucker \& Hancock, 2010) and the support of life (Dietrich \& Perron, 2006). For our sustainable cohabitation in the world, we need to understand how landscapes 
shape themselves, which in turn requires a clear understanding of sediment generation and its subsequent transport and dispersal.

Understanding of sediment movement over time and space requires quantitative tools; these can be divided into sediment tracers, which follow sediment pathways and capturing their journey from source to sink, and sediment fingerprinting, which views sediment in the sink with an upstream focus to infer the source or provenance (following definitions and review by Koiter et al., 2013; see Table 1 for definitions used in this study). Sediment tracing can be further divided into methods that examine the dispersion of individual grains (e.g., Bradley, 2017) and into methods that quantify bulk fluxes of sediment (Belmont et al., 2014). Likewise, sediment fingerprinting can be subdivided into geographic fingerprinting (source location) and geomorphic fingerprinting (source landforms, e.g., upland soils, channel banks, and landslides; Belmont et al., 2014). Each of these subdivisions serves to answer a specific family of questions concerning sediment transport or sourcing, and further development of new methods is necessary for continued progress (Walling, 2013).

A potential expansion of methods in sediment tracing and fingerprinting lies within trapped charge phenomena such as optically stimulated luminescence (OSL), infrared stimulated luminescence (IRSL), and thermoluminescence (TL). OSL, IRSL, and TL, hereafter referred to as "luminescence," are light emissions from minerals where the magnitude and characteristics depend on the grain's crystallographic defects, previous sunlight-exposure, and irradiation history. As such, luminescence can be used to determine the time since sunlight exposure, which serves as the basis for many widely used geochronologic techniques for Quaternary-age deposits (Rhodes, 2011). However, the sensitivity of luminescence to sunlight holds another key advantage in that grains in different geomorphic systems or even at different locations within the same geomorphic system, experience different levels of light exposure. This phenomenon leads to spatial variations in the magnitude of luminescence, raising the possibility of quantifying sediment transport rates via measurements of luminescence. In addition, the intrinsic behavior of luminescence itself can be a function of source geology, presenting a potential tool for downstream sediment tracing and provenance reconstruction.

The use of luminescence to directly quantify geomorphic processes is an important research frontier (Heimsath \& Ehlers, 2005). The potential applications of luminescence are rapidly expanding to provide new quantitative insights into geomorphic processes such as exhumation using OSL (Herman et al., 2010) and TL (Biswas et al., 2018; Brown et al., 2017), soil mixing and transport (Furbish, Schumer, et al., 2018; Reimann et al., 2017), surface exposure (Sohbati et al., 2012) and surface erosion (Sohbati et al., 2018; Guralnik \& Sohbati, 2019), source area weathering and erosion (Haddadchi et al., 2016; Sawakuchi et al., 2018), fluvial sediment transport (Gray et al., 2018; McGuire \& Rhodes, 2015a), and coastal sediment transport (Ahmed et al., 2013; Reimann et al., 2015). Luminescence can provide quantitative sediment transport data from source to sink (Figure 1) including the identification of provenance (Figure 2). Here, we review the current state of luminescence as a sediment tracer with a view toward bringing together the existing disparate literature on the subject and to evaluate its potential for tracing sediment.

We have organized this paper by first discussing the physical basis for luminescence as a sediment tracer in geomorphic environments. Next, we review and summarize previous applications of luminescence sediment tracing toward quantifying sediment transport rates, followed by a review and synthesis of luminescence as a provenance tool. We focus on studies that directly use luminescence intensities, equivalent doses, or luminescence characteristics to infer sediment transport or depositional processes as opposed to dating of deposits.

\section{Overview of Sediment Tracing and Fingerprinting}

Determining provenance via mineralogical fingerprinting dates back to the late nineteenth century (Weltje \& von Eynatten, 2004). The mid-1970s saw an expansion of analytical capabilities including geochemical techniques (see Walling, 2013, for a comprehensive review). Similarly, sediment tracing has roots extending to the early twentieth century (Hassan \& Ergenzinger, 2003) with demonstrations of the use of painted tracers in a flume by Einstein (1937) and in natural rivers by Takayama (1965) and Leopold (1966). Over time, sediment tracing has continually expanded with the development of new techniques (see reviews by Hassan \& Ergenzinger, 2003, and Gellis \& Walling, 2011). 
Table 1

List of Terms Used in This Paper and Their Definitions

Term

Definition

Bleached sediment

Bleaching

Bleaching rate $\sigma \varphi$

Characteristic dose, $D_{0}$

Dose rate, $D_{R}$

Electron trap

Hole

IRSL

LM-OSL

Luminescence

Luminescence characteristics

Luminescence decay curve

Luminescence growth (dose response) curve

Luminescence sensitivity

OSL

Quartz OSL components

Saturation or saturated luminescence

Sediment fingerprinting

Sediment provenance

Sediment tracer

TL

Unbleached or partially bleached sediment
Sediment that has had complete, or near complete removal of luminescence.

A term referring to the removal of luminescence by some process such as sunlight exposure.

How fast luminescence is removed by sunlight exposure for a specific electron trap. Set by the ambient photon flux $(\varphi)$ and the photoionization cross section $(\sigma)$, effectively a "bleachability" or a ability of a trap to capture a photon.

The amount of radiation dose needed to increase the luminescence from 0 to (1-1/e; approximately $63 \%)$ of the saturated luminescence value. Units of Joules per kilogram, also units of Gray after physicist Louis Harold Gray.

The natural background rate of ionizing irradiation that regenerates luminescence in sediment grains over time while buried. Typically created by the presence of potassium, uranium, thorium, and other elements in addition to cosmic radiation. Units of Joules per kilogram per year.

A defect in the crystal lattice of minerals that creates a meta-stable energy state capable of storing negative charge.

A location of positive charge available to combine with electrons.

Infrared stimulated luminescence. Luminescence that is produced when a material (such as a grain of sand) is exposed to infrared light. Typically measured on feldspar and usually absent in quartz.

Linearly-modulated optically stimulated luminescence. Luminescence that is produced when a material (such as a grain of sand) is exposed to optical light that is linearly increased in intensity over the measurement interval. Can resolve components with different bleachabilities and stabilities over time.

A grouped term referring to trapped charge phenomena such as optically stimulated luminescence, infrared stimulated luminescence, and thermoluminescence.

Properties of the luminescence of sand grains such as sensitivity, characteristic dose, ratios of LM-OSL components.

On exposure to light, luminescence is generated, but then quickly depletes in an exponential or power law fashion. The graph of luminescence (OSL or IRSL) versus exposure time is referred to as a decay curve.

A curve describing the relation between the luminescence and absorbed radiation dose.

The amount of luminescence generated per unit mass and per unit absorbed radiation dose. Can be specific to individual quartz OSL components.

Optically stimulated luminescence. Luminescence that is produced when a material (such as a grain of sand) is exposed to optical light. Typically measured from quartz, but can also be measured in feldspar.

Quartz OSL appears as the sum of multiple exponential decay curves with varying decay rates. Typically defined as "fast," "medium," and "slow," components depending on how fast the component decays with stimulation light. Can be best observed with LM-OSL.

The point at which all luminescence-generating electron traps are filled and no further radiation exposure will increase the measurable luminescence.

A technique of characterizing sediment to determine sediment source area or provenance. Generally, looks in an upstream direction. A classic example is locating unique minerals that indicate erosion of a geologic unit.

A location of a source of sediment. Can refer to geographic locations such as outcrops or geomorphic processes such as landslides.

A property or characteristic of sediment used to distinguish individual grains or populations of grains to infer their individual or collective movement. Generally, refers to downstream observations of sediment transport. A classic example is painting sand grains and observing their movement downstream.

Thermoluminescence or thermally stimulated luminescence. Luminescence that is produced when a material (such as a grain of sand) is heated.

Sediment that has either never had luminescence removed, or still contains a high amount of luminescence from insufficient exposure to a bleaching agent such as sunlight.

Abbreviations: IRSL, infrared stimulated luminescence; LM-OSL, linearly modulated optically stimulated luminescence; OSL, optically stimulated luminescence; TL, thermoluminescence.

The popularity of sediment tracing and fingerprinting stems from its applicability to a wide variety of research questions. Hassan and Ergenzinger (2003) note that tracers can be used to provide information on the rate and direction of sediment transport, step lengths and residence time of particles, virtual rate of sediment movement, impact of sedimentological environment on distance of movement, sediment sources and depositional areas, and dispersion of contaminants; in addition, tracers can help address other potential research questions such as those involving soil erosion and accumulation (Ritchie \& McHenry, 1990). Similarly, reconstructing sediment provenance via fingerprinting also has the potential to answer a variety of research questions. These questions range from how and where landscapes produce sediment (Guzman et al., 2013; Koiter et al., 2013; Zhang et al., 2001), how sediment routing systems exchange and store 


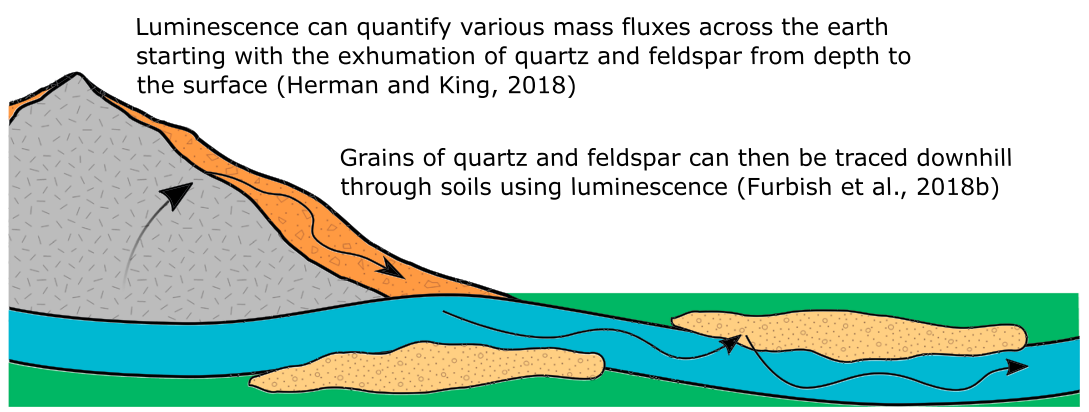

On entrainment in rivers, virtual velocities can potentially be estimated (Gray et al.,2018) and luminescence characteristics used for provenance (Sawakuchi et al., 2018).

Figure 1. Illustration of how luminescence of mineral grains can quantify the movement of mass across the Earth's surface.

sediment (Belmont et al., 2014; Pizzuto et al., 2014), and how various geomorphic processes modulate sedimentary fluxes (Walling, 2013). In turn, the answers to these questions have implications for catchment management and restoration (Collins et al., 2017; Mukundan et al., 2012); practical applications such as reservoir design, land-use planning, and archeological reconstructions (Davis \& Fox, 2009; Gellis \& Walling, 2011; Hassan, 1978; Koiter et al., 2013); natural and human-induced landscape evolution (Syvitski et al., 2005; Tucker \& Hancock, 2010); and inferring paleoclimatic and tectonic changes from the stratigraphic record (Weltje \& von Eynatten, 2004).

Just as the questions that sediment tracing and fingerprinting can answer are diverse, sediment tracer and fingerprinting methods vary considerably (Guzman et al., 2013; Koiter et al., 2013). Examples of methods include fallout radionuclides ${ }^{137} \mathrm{Cs},{ }^{210} \mathrm{~Pb}$, and ${ }^{7} \mathrm{Be}$ (Kaste et al., 2007; Mabit et al., 2014; Walling, 2013); cosmogenic isotopes (Lauer \& Willenbring, 2010); elemental concentrations (Govin et al., 2012; Viers et al.,

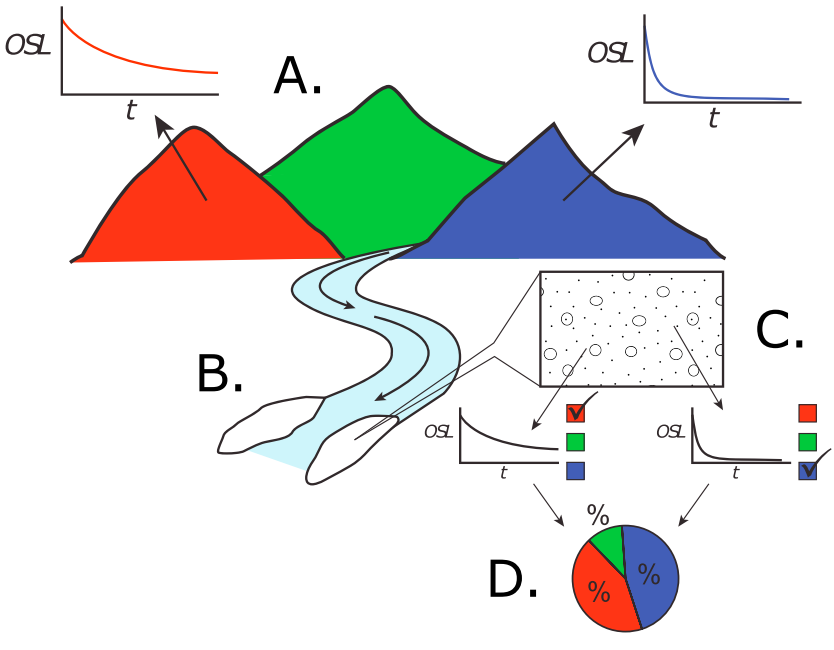

Figure 2. Basic conception of luminescence provenance methods. (a) Various source areas contribute with sediment in various proportions. (b) This sediment is amalgamated by sediment transport processes downstream. (c) Sampling of sediment and measurement of the luminescence characteristics of polymineral mixtures or concentrates of single mineral type allows us to classify source terrain. For example, one source may contain more quartz than another. (d) Synthesis of the data provides insight into the contributions of various terrains. Measurements in individual grains or unmixing statistical models allow to quantify the contribution of each sediment source. OSL: optically stimulated luminescence, $t=$ time.
2008); mineralogy (Morton, 1985); magnetic properties (Just et al., 2012; Milan \& Large, 2014); radio-frequency identification tags (Bradley \& Tucker, 2012; Hassan \& Ergenzinger, 2003); paint and fluorescent dyes (Hassan \& Ergenzinger, 2003); and contaminant adsorption (Pizzuto et al., 2014). Generally, sediment tracing and provenance studies are cost-effective solutions to research questions (Hassan \& Ergenzinger, 2003). Field work involves collection of sediment from field sites such as river channels and upland sources. Quantitative analysis and statistical operations can then quantify the portion of sediment derived from a given source (Gellis \& Walling, 2011; Weltje, 1997). In addition, tracing and fingerprinting methods can be applied to sediment cores to evaluate geomorphic and climatic changes over time (e.g., Mulitza et al., 2010).

Sediment tracing and fingerprinting methods are not without challenges, and several issues remain as outstanding problems. One challenge is the "black box" of sediment transport or the assumption of a direct link between sinks and sources without any transformation of the sediment (Koiter et al., 2013). In geomorphic systems, a wide variety of processes influence the motions of sediment, and during transport, sediment may undergo chemical, biological, and physical transformations including changes in particle size (Laceby et al., 2017). In addition, sediment can be stored in geomorphic compartments, such as floodplains, for long periods of time during transit from source to sink (Belmont et al., 2014; Pizzuto et al., 2014; Walling, 2013). This storage can potentially bias the record as can heterogeneous sediment mixing (Haddadchi et al., 2013). How these various geomorphic processes can bias sediment fingerprinting remains an open and potentially site-specific research question. Another 
potential issue lies in a lack of understanding of how nonconservative tracers may change during transport, such as the decay of isotopes or wearing of painted grains. However, the solution to these problems may lie in the use of multiple tracers at a field site and the development of new alternative tracers (Walling, 2013). Because of the wide variety of tracer applications, improving our ability to trace sediment is an important step in solving critical scientific and applied problems.

A potential expansion in sediment tracing and fingerprinting lies in using trapped charge phenomena such as luminescence. A key advantage of luminescence is its applicability to fine and very fine sand (63- to $250-\mu \mathrm{m}$ diameter grains) as well as to silt (4- to 62- $\mu \mathrm{m}$ diameter grains). As noted by Zhang et al. (2001) and Guzman et al. (2013): “... the ideal tracer would have the following characteristics: (a) strong binding to soil particles or ready incorporation into soil aggregates, (b) high analytical sensitivity, (c) easy and inexpensive to quantify, (d) low background soil concentration, (e) no interference with sediment transport, (f) low plant uptake, (g) environmentally benign, and (h) available in variants with similar, but distinguishable, physicochemical properties for multiple tracking." Luminescence and luminescence characteristics of minerals such as quartz and feldspar arguably fit many or all of these characteristics. No modification of the sediment grain, such as painting or tagging a grain, is needed to measure luminescence or its properties, meaning that sediment transport can occur unhindered. There is no potential for contamination of plants or the environment, as could occur with artificial materials or artificial radioactive elements. Luminescence measurements are made on natural sediment, sampling is conducted with non-toxic products, and there is no introduction of artificial material to the natural system being sampled. Furthermore, luminescence encompasses a wide range of variants that can be measured with high analytical sensitivity at a low cost with minimal sample preparation. In particular, the use of quartz luminescence has the benefit of low weathering rates and high preservation potential over long timescales.

\section{Physical Basis of Luminescence as a Sediment Tracer and Provenance Tool}

\subsection{The Physics of Luminescence}

Luminescence, here OSL, IRSL, and TL, originates from charge populations in the crystal lattice of minerals (Huntley et al., 1985; Aitken, 1998; Yukihara \& McKeever, 2011). At the atomic scale, ionizing radiation in the kilo-electrovolt to mega-electrovolt range interacts with atoms and creates free electrons and holes (locations of negative and positive excess charge in the mineral lattice, respectively). A tiny fraction of these free charges, including electrons in the conduction band and holes in the valence band, can occupy allowed energy levels created by the presence of structural defects (such as atomic vacancies) or atomic impurities. These energy levels are commonly known as "traps," and they form metastable states once they capture electrons or holes. Because such traps are finite in concentration, they fill up over time until the system reaches a "saturated" state in which no more free electrons or holes can be stored.

The reverse process of trap emptying requires external energy such as heat or light, which leads to detrapping and the return, or recombination, of electrons with the trapped holes (Figure 3). This return of the system to its prior state (before exposure to ionizing radiation) is accompanied by light emission, or luminescence, due to the release of energy stored in the metastable states. Thus, luminescence is a proxy for the concentration of trapped electrons/hole pairs. For luminescence measurements applied to dating, one typically targets those traps that are thermally stable on the time scales of millions of years. Electrons in these deep traps can be released by the application of visible to near infrared wavelengths or by heating to several hundreds of degrees. Specific terms refer to the use of the laboratory stimulation method to produce luminescence. For example, OSL or IRSL refer to the use of stimulation light in the optical range to near-infrared range (400- to $900-\mathrm{nm}$ wavelengths), and TL refers to the use of heat to produce luminescence. These various forms of luminescence are typically measured with a luminescence reader consisting of light emitting diodes or lasers, photomultiplier tube, heating element, and radiation source (Bøtter-Jensen et al., 2003).

The intensity of luminescence emission is related to the concentration of trapped charge population, both electrons and holes, and the efficiency of radiative recombination (how easily an electron can access a hole; Yukihara \& McKeever, 2011). In nature, a growth in trapped charge due to exposure to natural ionizing radiation (e.g., from decay of radioactive isotopes during burial) leads to an increase in the luminescence. Conversely, a decrease in trapped electron population due to exposure to daylight (e.g., during weathering 
A) Crystal Lattice Defects

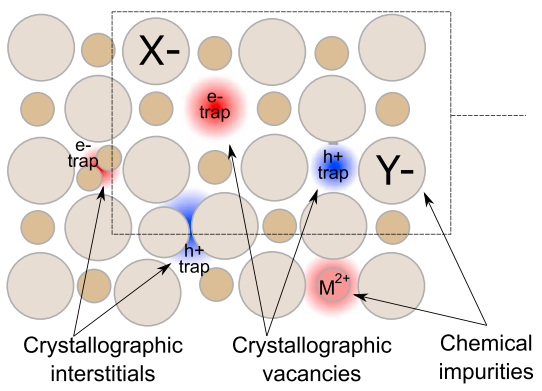

B) lonizing Irradiation

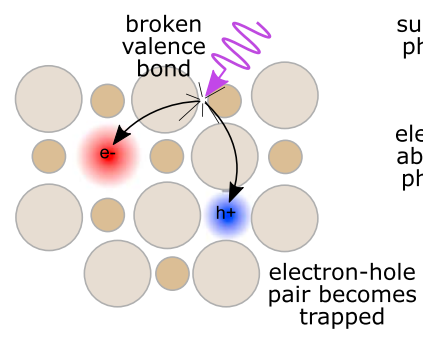

C) Sunlight Exposure

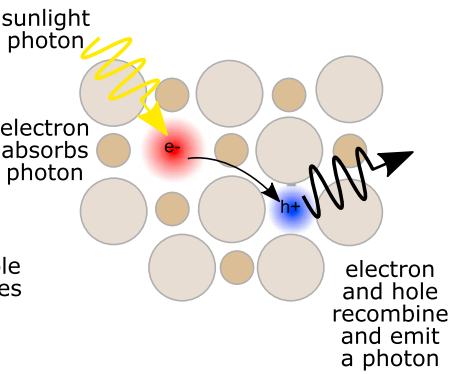

Figure 3. Simplified schematic of electron-hole pair creation and recombination, adapted from King et al. (2016). (a) The crystal lattice of minerals often contains defects that can trap electrons (e $\left.\mathrm{e}^{-}\right)$and holes (centers of positive charge: $\mathrm{h}^{+}$). This schematic shows an alkali-halide-like lattice with defects such as vacancies, interstitials, and impurities. (b) Ionizing radiation from decaying radioactive elements (e.g., potassium, uranium, and thorium) creates electron-hole pairs by interacting with the lattice. (c) If an electron receives enough energy to escape, say via absorbing a photon of sunlight, it can recombine with a hole, emitting a photon in the process. We can measure these photons as luminescence and treat the number of photons as a proxy for the trapped charge in a mineral.

and transportation) leads to a decrease in the luminescence signal. Thus, luminescence is a dynamic quantity that can grow and decay with time depending on the history of burial or exposure (Figure 4). In turn, the history of burial and exposure is a function of geomorphic environment and sediment transport conditions and the available accommodation space (Jain et al., 2004).

For the same absorbed energy from ionizing radiation, different samples can emit different intensities of luminescence. This observation is referred to as the luminescence sensitivity of the sample, effectively the luminescence produced per unit mass per unit dose of radiation. Sensitivity varies across samples because the concentration of electron trapping states, the ratio of luminescent/nonluminescence radiative recombination centers, and trapping competition effects in a crystal depend upon the sample's geochemistry and geological history. For siliciclastic sediments, quartz grains originating from different source rocks can have different luminescence behavior due to the temperature and pressure conditions of crystallization (e.g., Guralnik, Ankjærgaard Jain, et al., 2015; Guralnik, Jain, et al., 2015), although quartz from crystalline rocks typically has low luminescence sensitivity (Moska \&

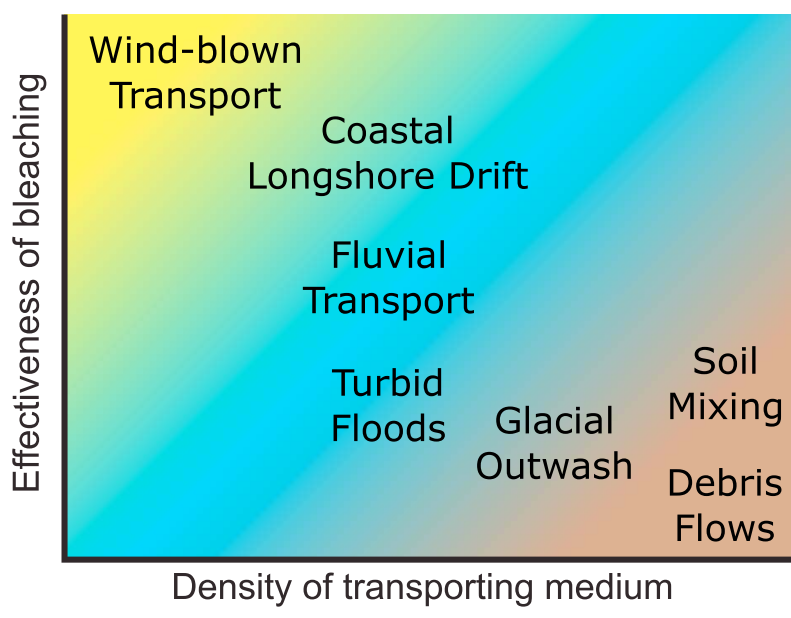

Figure 4. Demonstration of the bleaching (equation (2)) and regenerating (equation (1)) nature of luminescence of a single sediment grain during sediment transport. Note that the regeneration phases are on the order of tens to thousands of years, whereas the decay phases are hypothesized to operate on seconds to days or weeks. The average luminescence of thousands of grains or luminescence characteristics of single grains may give insight into the transport mechanisms and rates (Gray et al., 2017). For color references, the reader is referred to the online color version of the article. Murray, 2006). Because of this link with geological source, the sensitivity of quartz grains in a sediment sample would be a function of the sample's provenance. However, further studies indicate that major changes in the luminescence sensitivity of quartz occur after quartz crystals from rocks are converted into sediment grains (Sawakuchi et al., 2011), although this requires further investigation. Thus, the sensitivity of a sample can change based on the previous number of cycles of deposition and erosion, which promote successive events of burial irradiation and sunlight exposure (Moska \& Murray, 2006; Pietsch et al., 2008). Generally, the sensitivity of quartz grains increases with progressive deposition-erosion cycles, but this is not always the case, because some single grains present stable sensitivity after being submitted to irradiationillumination cycles (Pietsch et al., 2008).

\subsection{Functional Forms of Luminescence Growth and Decay}

The physics of luminescence can be modeled with equations for both luminescence growth and decay. When buried in a deposit and subject to background ionizing radiation, the luminescence of a sand or silt-sized grain or multigrain aliquot grows following, in its simplest functional form, a saturating exponential form: 


$$
L(t)=L_{\mathrm{sat}}\left(1-\exp \left(-\frac{D_{R}}{D_{0}} t\right)\right)
$$

where $L$ (dimensionless) is the luminescence as a proxy for trapped electron population (after sensitivitycorrection, a form of normalization), $t$ is time (s), $L_{\text {sat }}$ (dimensionless) is the luminescence at "saturation" (i.e., when: $t \rightarrow \infty), D_{R}$ is the environmental dose rate (Gy/s), and $D_{0}$ is the characteristic dose (Gy), a parameter defining the amount of dose needed to reach 63\% of $L_{\text {sat }}$. Equation (1) (Wintle \& Murray, 2006) shows how latent luminescence will increase with storage, while a grain is buried and exposed to ionizing radiation. Typically, $D_{0}$ values for single grains of quartz range from $\sim 10$ to $100 \mathrm{~Gy}$ (Duller, 2012), but large variation can be found in the literature (Singarayer \& Bailey, 2003). Values of $D_{0}$ may be due to source lithology, but further evidence is needed to confirm this (Lowick et al., 2010). Recently, Timar-Gabor et al. (2017) demonstrated that an inverse square relationship exists between quartz grain size and $D_{0}\left(D_{0}=\frac{k}{\sqrt{\delta}}\right.$, where is grain size and $k$ is a constant).

The luminescence of a grain decreases when the sample is exposed to sunlight or heat, example daylight exposure during weathering/transport or heating. A simplified way to describe this decrease in luminescence, following first order kinetics, is an exponential loss with exposure time:

$$
L(t)=L_{0} \exp (-\lambda t)
$$

where,

$$
\lambda= \begin{cases}\sigma \varphi & \text { for light exposure } \\ s e^{-E / k_{B} T} & \text { for heat exposure }\end{cases}
$$

wherein $L_{0}$ is the luminescence when $(t=0)$ at the onset of exposure and $\sigma\left(\mathrm{cm}^{2}\right)$ is a probability that a trapped electron will interact with a photon from given light flux $\varphi\left(\mathrm{cm}^{2} / \mathrm{s}\right)$. Similarly, in case of heating, $s$ $\left(\mathrm{s}^{-1}\right)$ is an electron attempt-to-escape-the-trap frequency, $E(\mathrm{eV})$ is the activation energy necessary to overcome the trap potential, $k_{B}$ is Boltzmann's constant, and $T\left({ }^{\circ} \mathrm{K}\right)$ is temperature (McKeever \& Chen, 1997). Equations (1) and (2) represent the ideal behavior of luminescence as a function of environmental variables (background dose rate $D_{R}$ and light flux $\varphi$ ) and intrinsic luminescence variables (characteristic dose $D_{0}$ and "bleachability" governed by $\sigma$ ). Different luminescence signals such as OSL, IRSL, TL, and their derivative signals have varying levels of complexity in their underlying equations and are often approximated as powerlaws, sums of exponential functions, or systems of differential equations (e.g., Chen \& Pagonis, 2011; Guralnik, Li, et al., 2015; Huntley, 2006; Jain et al., 2012, 2015). The above equations (1) and (2) have mainly been found to be appropriate for describing the luminescence behavior of the fast OSL component of quartz (Jain et al., 2003; Jain, Murray, et al., 2005; Singarayer \& Bailey, 2003). The kinetics of other luminescence systems are more complex and require further understanding. In general, the relationship between light and heat is complex and it is well known from the laboratory experiments that ambient temperature governs the optical depletion rate (Bøtter-Jensen et al., 2003; Jain \& Ankjærgaard, 2011; Wintle \& Murray, 1999). The model equations describing the growth and depletion of a certain luminescence signal set the basis for techniques such as thermochronology and/or thermometry (e.g., Brown et al., 2017; Rengers et al., 2017; Spencer \& Sanderson, 1994), exposure and erosion (Sohbati et al., 2018), and sediment transport (McGuire \& Rhodes, 2015a).

3.2.1. Quartz OSL Components

In the case of quartz OSL, the luminescence emitted during sediment bleaching appears as a sum of a discrete exponential decays (Bailey et al., 1997; Jain et al., 2003; Singarayer et al., 2003) corresponding to different traps. These exponential decays are termed as "fast," "medium," and "slow" based on the relative rate of decay (Bailey et al., 1997). For example, the total OSL can be described as a sum of components:

$$
L_{\text {total }}(t)=L_{0 \text { fast }} \exp \left(-\sigma_{\text {fast }} \varphi t\right)+L_{0 \text { medium }} \exp \left(-\sigma_{\text {medium }} \varphi t\right)+L_{0 \text { slow }} \exp \left(-\sigma_{\text {slow }} \varphi t\right)
$$

with the subscripts indicating the contributions of each component, which has the form of equation (2). Similarly, the growth of each component can be described by a similar summation of the right-hand side term in equation (1). The presence and the relative amounts of luminescence produced by each component per unit dose is a function of source lithology and transport history (Choi et al., 2006; Haddadchi et al., 2016; Kuhns et al., 2000; Jain et al., 2003; Jeong \& Choi, 2012; Sawakuchi et al., 2011; Singarayer et al., 2005; 
Tsukamoto et al., 2011). Quartz extracted directly from igneous or metamorphic bedrock can be dominated by the medium and slow components (Jeong \& Choi, 2012), whereas sedimentary transport appears to increase the sensitivity of the fast component (Haddadchi et al., 2016; Jeong \& Choi, 2012; Sawakuchi et al., 2011). This dependence on source lithology provides the basis for OSL components as a provenance tool (Haddadchi et al., 2016), as discussed in detail in further sections. Individual components can be observed more easily using linearly modulated optically stimulated luminescence (LM-OSL; Bulur, 1996), where the stimulating light flux is linearly increased in intensity over the measurement interval. The linear increase in stimulating light flux causes the separate OSL components to peak at different times during measurement and allows them to be easily visually separated.

\section{Luminescence and Its Characteristics in Geomorphic Environments}

In the following subsections, we summarize knowledge about how luminescence and its characteristics may change in geomorphic systems. We broadly discuss the 4- to $250-\mu \mathrm{m}$ grain-size range (very fine silt to fine sand), although these concepts may also apply to a broader range of sediment grain sizes such as cobbles (e.g., Liu et al., 2018).

The dynamic nature of luminescence in geomorphic environments is the key strength that differentiates luminescence from other tracer methods. As grains of sand migrate through geomorphic systems, such as rivers, hillslopes, and aeolian dunes, the grains will experience varying levels of light exposure (luminescence removal) and varying time spent in burial (luminescence regeneration). Broadly, the level of light exposure and luminescence removal for a sediment grain in a geomorphic system depends on the transporting medium and mechanism (Figure 5). In contrast, luminescence regeneration is dependent on the inherent storage times within a geomorphic system, and on the concentrations of radioactive elements in sediments (such as uranium, thorium, potassium, and others) and exposure to cosmic radiation (Prescott \& Hutton, 1994). This means that the luminescence of sediment grains at any given point in space or time will reflect the rates and mechanism of the sediment transport process (Figures 4 and 5) and the ambient sediment environment. To our knowledge, no other sediment tracer has this specific sensitivity to storage time and sunlight exposure, although some tracers such as beryllium-7 $\left({ }^{7} \mathrm{Be}\right)$ and excess lead-210 $\left({ }^{210} \mathrm{~Pb}\right.$ ex $)$ can be affected by burial (Gellis et al., 2018; Koiter et al., 2013). Thus, luminescence has unique potential to uncover new information on earth surface systems, possibly recording rates and mechanisms of sediment transport.

\subsection{Luminescence Bleaching in Different Environments}

To understand how to use luminescence as a sediment tracer, we must understand how sunlight exposure removes, or "bleaches" (Wintle, 1981), luminescence in geomorphic environments. To gain this understanding, we must determine (1) how sediment grains typically get exposed to sunlight, (2) what is the spatial pattern of luminescence at different scales, and (3) how do spatial patterns of luminescence vary over geomorphic timescales. Answers to these questions for a specific geomorphic system determine our ability to decipher transport histories recorded in luminescence.

First, how are sediment grains typically exposed to sunlight in geomorphic environments and how does luminescence bleaching occur as a result? Bleaching rates can vary by orders of magnitude depending on the light flux. The time needed to fully bleach a luminescence signal ranges from seconds in direct sunlight to months and significantly longer in turbid water (Gemmell, 1985; Gray et al., 2018). Bleaching can occur prior to transport, during transport (as for example when a grain is suspended in a fluid flow), and/or following deposition on a surface, where a stationary grain can receive prolonged sunlight exposure (Gray \& Mahan, 2015). The processes that dominate depend on the local geomorphic environment. For example, grains in a soil may only become bleached by direct surface exposure, when grains are brought to the top of the soil column by various mixing processes (Heimsath et al., 2002). In contrast, some systems may bleach sediment by more than one process. For example, sediment in a deltaic system may experience bleaching by sunlight exposure of grains deposited on subaerial surfaces such as bars and by in-transport bleaching when grains are suspended in a flow (Chamberlain et al., 2017; Chamberlain, Törnqvist, et al., 2018, Chamberlain, Wallinga, et al., 2018). Understanding how bleaching occurs serves to define what information one can obtain from the luminescence signal. In the case of soil, the interpretation of luminescence is straightforward: bleaching at the surface and mixing of soil causes the luminescence to record information on the soil mixing rate (Reimann et al., 2017). In the case of river systems, interpretation of the luminescence is river- 


\section{Schematic luminescence of a single sand grain in idealized geomorphic transport}

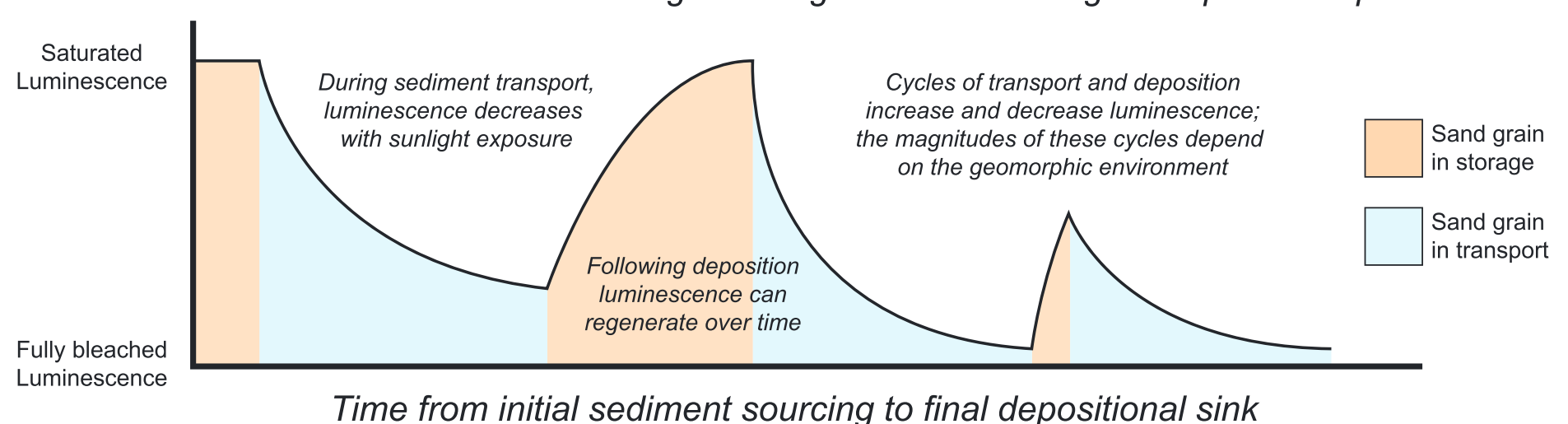

Figure 5. Conceptual graph of bleaching effectiveness (ability to completely remove an inherited dose related to burial) versus density of transporting medium. Density is loosely interpreted as low for air (e.g., aeolian sediment) and higher for water (e.g., fluvial), and highest when sediment concentrations are high or sediment is part of the flow (e.g., glacial outwash and debris flows). Generally, higher density flows lead to lower bleaching effectiveness.

specific. Depending on the bleaching mechanism, the luminescence may record the time spent in transport, the frequency of flooding events, and time in storage (Gray \& Mahan, 2015).

In cases of systems with multiple bleaching processes, interpreting luminescence requires one to determine which process is dominant (Gray et al., 2017). River systems serve as a useful example, as the relative influence of sunlight exposure on surfaces, such as bars, versus in-transport bleaching, is not yet well known (Gray \& Mahan, 2015). The uncertainty stems from the number of variables that control both processes (Cunningham, Wallinga, et al., 2015; Jain et al., 2004). For in-transport bleaching, subaqueous bleaching rates depend on time of day and on water turbidity, which increases light attenuation and modifies the impinging wavelengths of sunlight, and reduces the rate of luminescence bleaching (Berger, 1990; Ditlefsen, 1992; Kars et al., 2014; Rendell et al., 1994; Sanderson et al., 2007). Water turbidity is itself controlled by sediment supply and river hydrology. For surface bleaching, key variables include the number of flood events that rework sediment, the typical depths of scour versus light penetrative depths, and the surface area of sunlight-exposed deposits set by channel and floodplain geometry. Because these variables range widely among different fluvial systems, the relative influence of in-transport versus surface bleaching is likely to vary from river to river and may be flood specific (Gray et al., 2017).

While we are not yet certain how bleaching generally occurs, sparse research provides some insight. Laboratory flume experiments indicate that in-transport bleaching of TL is virtually negligible (Gemmell, 1985). On the other hand, it is not clear whether the faster-to-bleach OSL/IRSL signals also bleach slowly in flumes or how flume studies scale up to natural rivers. Field-based approaches offer additional insight. For example, in a flash-flood driven system, such as the Mojave River in southern California, USA (McGuire \& Rhodes, 2015a, 2015b), bleaching should be dominated by surface exposure as the river's flood waters are extremely turbid with very limited sunlight exposure (Porat et al., 2001). However, McGuire and Rhodes (2015b) found that single-grain distributions did not match predictions of a pure surface bleaching model for the Mojave River. The authors suggest that both surface bleaching and in-transport bleaching are occurring even in this turbid flash flood driven system. In contrast, Gray et al. (2017) argued that bleaching via surface exposure for some river systems may be less important than in-channel bleaching as the fraction of sediment exposed at the surface is small when compared to the overall volume moved during the floods that move most of the sediment. While it is not immediately clear when and where in-transport or surface bleaching is dominant, answers to this problem may lie in examining changes in sensitivity as a function of reworking cycles (Gliganic et al., 2017; Pietsch et al., 2008), the uniformity of bleaching in single grain distributions (Arnold et al., 2007; Duller, 2008; Rittenour, 2008), and/or the ratios of easy-to-bleach versus hardto-bleach luminescence signals (Fiebig \& Preusser, 2007; Murray et al., 2012; Singarayer et al., 2005).

Despite some uncertainty in the mechanisms of bleaching, researchers have noticed general landscape-scale spatial patterns of bleaching in geomorphic environments. Generally, luminescence has been observed to 
decrease with the transport distance (1-100 km; Gliganic et al., 2017; Hu et al., 2010; McGuire \& Rhodes, 2015a; Stokes et al., 2001). In an idealized landscape with idealized luminescence, the bleaching rates are constant throughout, such that the luminescence lost per unit transport distance is a function of drainage area. In such a case, stream junctions deliver sediment with volume and luminescence proportional to the upstream drainage area and the theoretical result is a power-law decrease in luminescence downstream (Gray et al., 2017; Gray et al., 2018). However, nature is heterogeneous and the downstream decrease can change to an increase if enough unbleached sediment is delivered to the channel relative to the current sediment load (Gray et al., 2017; McGuire \& Rhodes, 2015a). Delivery of unbleached sediment can occur if a tributary is less effective in bleaching than the main channel, say due to enhanced sediment load (Gray et al., 2017; Li et al., 2018). Other examples include entrainment of unbleached sediment by river channel incision (Muñoz-Salinas et al., 2012), lateral channel erosion due to river meander, and variability in soil erosion rates (Gray et al., 2018). Broadly, the spatial patterns of luminescence within a river system, or across river systems, may have the potential to give insights into flood dynamics (Knight \& Evans, 2018) and into landscape dynamics (Muñoz-Salinas, Castillo, Caballero, et al., 2017; Portenga et al., 2016). However, it is not yet known how specific geomorphic processes result in specific changes in luminescence such that we can quantitatively predict their effects.

In addition to large-scale spatial patterns, researchers have documented variability in luminescence bleaching at small spatial scales ( $1 \mathrm{~cm}-1 \mathrm{~m}$ ). King et al. (2013), King, Robinson, et al., (2014) observed that residual luminescence, as apparent ages, can vary notably between glacial and periglacial geomorphic features. Variability in apparent age is also large within glaciofluvial deposits (King, Sanderson, et al., 2014). Cunningham, Wallinga, et al., (2015), Cunningham, Evans, et al., (2015) noted that there appears to be a correlation between bleaching and the discharge of flood that deposited sediment in a bedrock river setting, with large floods appearing more likely to deposit unbleached sediment. At the smallest spatial scale, researchers have noted differences in bleaching of fluvial deposits as a function of grain size, with coarser grains (180-250 $\mu \mathrm{m}$ ) being better bleached (Alexanderson, 2007; Hu et al., 2010; Olley et al., 1998; Rittenour, 2008; Tóth et al., 2017; Truelsen \& Wallinga, 2003; Vandenberghe et al., 2007). This observation is puzzling, as finer grains should spend more time in suspension and consequently are exposed to light for a longer duration than are coarser grains (Gray \& Mahan, 2015). However, differences in bleaching by grain size are not ubiquitous, as some researchers have found that finer grains have lower residual doses (Fuchs et al., 2005; Hu et al., 2010; Schielein \& Lomax, 2013) and there are cases where all grain sizes seem adequately bleached (e.g., Chamberlain \& Wallinga, 2018)

There are some potential hypotheses to explain the differences in residual dose between grain sizes. One is that different grain sizes have different rates of exchange between a channel and floodplain (Lauer \& Willenbring, 2010) such that the total time spent regenerating luminescence during storage varies for sediments of different grain sizes, which leads to different levels of luminescence in channel sediment. Another hypothesis is that the physics of vibration induced by fluid action on deposits causes coarse grains to rise to the surface of deposits, leading to sunlight exposure at the surface.

In addition to spatial variability, temporal changes in channel sediment luminescence can hint at geomorphic process. Gemmell (1997) gives a key example through measurements of the TL of suspended polymineral sediment over the course of a discharge event in an alpine stream. Gemmell (1997) found that average TL intensity was low at the beginning of the event but increased in intensity with duration of the event. Gemmell (1997) reasoned that the TL magnitude reflected the initial entrainment of highly bleached surface-exposed sediment by the stormflow followed by scouring of unbleached sediment in deposits and streambanks. TL signals in similar streams appear to show systematic fluctuations over time (Gemmell, 1994a, 1994b) such as diurnal fluctuations (Gemmell, 1997, 1999). This may be due to changes in bleaching rates between daylight and sunlight-diminished conditions (Lindvall et al., 2017). However, some systems may not show regular patterns (Gemmell, 1988). How and when luminescence in sediment changes remains an open research question that may offer insight into geomorphic processes.

Most research into bleaching has focused on river systems, but bleaching varies greatly by geomorphic environment (Alexanderson, 2007; Jaiswal et al., 2009; Przegiętka et al., 2016; Schielein \& Lomax, 2013; Weckwerth et al., 2012). For a given luminescence signal (OSL or IRSL), aeolian sediments often show lower residual doses (remnant luminescence) than other types of sediment (Buylaert et al., 2011; Singarayer et al., 
2005). In aeolian systems, bleaching tends to be more complete for individual grains presumably, because typical transport conditions in arid systems generally allow for less attenuated solar irradiance as compared to subaqueous transport (Wintle, 1993) and general greater mobility of wind-blown sediment allows long subaerial durations. In contrast, deltaic systems host highly variable bleaching conditions that probably reflect varying levels of water turbidity, sediment transport distances, and driving mechanisms such as streamflow and wave action, leading to large variation in the extent of bleaching (Chamberlain et al., 2017, Chamberlain, Törnqvist, et al., 2018). As with deltaic systems, coastal systems involve an ensemble of processes and factors, such as climate-modulated storm frequency and magnitude, that control wave action, which in turn controls sediment sourcing, transport, reworking, and light exposure (Fruergaard et al., 2015; López et al., 2018; Reimann et al., 2015; Sawakuchi et al., 2012; Zular et al., 2015). As such, incompletely bleached sediment is not uncommon in coastal systems (Richardson, 2001; Jacobs, 2008; Alexanderson \& Murray, 2012), although well-bleached samples can be found (Murray et al., 1995; Madsen \& Murray, 2009; Chamberlain, Wallinga, et al., 2018). Further inland, such as in mixing soils on hillslopes, bleaching occurs entirely by surface exposure where grains reach the surface via mixing processes and are then reburied (Reimann et al., 2017). Finally, in rare cases, bleaching can potentially occur via mechanisms such as pressure at the bottom of a glacier (Bateman et al., 2018). The wide variety in the extent of bleaching, and our limited understanding of the processes, shows that much is yet to be learned about the connections between luminescence and geomorphology.

\subsection{Patterns of Luminescence Regeneration in Different Environments}

To best utilize luminescence as a sediment tracer and fingerprinting tool for sediment grains, we must recognize the role of sediment storage during geomorphic transport. Sediment storage provides an opportunity for luminescence to regenerate and to undergo changes in luminescence characteristics such as sensitivity over many burial-erosion cycles (Pietsch et al., 2008). Sediment storage is a ubiquitous process. If one were to pick a grain of sediment at random, it is overwhelmingly more likely to find it in a stationary state and residing in a depositional feature, than to find it in a state of motion (Bradley \& Tucker, 2013; Meade, 2007). Furthermore, these stationary periods can range from seconds up to millions of years (Pizzuto et al., 2017) with the time spent in storage controlled by the type of geomorphic system (e.g., glacial, fluvial, aeolian) and the "connectivity" of different storage reservoirs (Hoffmann, 2015).

For context, luminescence regeneration during storage occurs over generally long timescales. As an example calculation, one can rewrite equation (1) as $t=-\left(D_{0} / D_{R}\right) \ln \left(1-L / L_{\mathrm{sat}}\right)$. The time $(t)$ needed to regenerate luminescence from $L=0$ to $L=0.99 L_{\text {sat }}$ requires approximately $70 \mathrm{kyr}$ (equation (1), approximate value for $D_{0}=47 \mathrm{~Gy}, D_{R}=3 \mathrm{~Gy} / \mathrm{kyr}$; Duller, 2012). The minimum resolvable timescale depends on the precision of a dose measurement and the sensitivity of the sample. A single day is unlikely to be resolvable with quartz OSL, whereas a thousand years is likely resolvable, and some highly sensitive samples can resolve tens to hundreds of years.

It is challenging to summarize all possible sediment storage systems and their effect on luminescence. However, some generalizations at various scales can be useful. At the landscape scale, it is useful to consider how various geomorphic processes and sedimentary reservoirs exchange sediment. For example, the production of sediment via soil forming processes on hillslopes is eventually delivered to river channels by gravitydriven transport (Roering et al., 1999). Depending on the transport capacity of the river channel, this sediment may become entrained into the flow and transported away (Hooke, 2003). Alternatively, the flux of sediment from hillslopes may overwhelm the river's capacity, thus retaining sediment in valley fill (Hooke, 2003). In the case of nonentrainment, sand grains have an opportunity to regenerate luminescence that may have been removed by prior sunlight exposure on hillslopes (Fuchs \& Lang, 2009). Here, it is the accommodation space of the river-hillslope system that allows for storage time and regeneration. These landscape-scale interactions between storage reservoirs are often referred to as "sediment connectivity," where systems with a high pass-through rate are considered high "connectivity" (Hoffmann, 2015). However, it is important to note that the connectivity of the example above is restricted to a timescale defined by the river and hillslope processes.

At scales smaller than landscape scale (less than tens of kilometers), the dynamics of individual processes have a control on sediment storage times. The extent of regeneration depends on probability distributions 
of resting times whose exact form is a function of the specific geomorphic process (Furbish et al., 2017). For example, a grain of sand travelling through a meandering river system may spend time between periods of in-channel motion and long-term storage in floodplain deposits. The probability distributions that define the grain's long-term resting time in such a meandering system can vary from exponential to power-law (Bradley et al., 2010; Bradley \& Tucker, 2013) with storage times ranging from years to hundreds of thousands of years. Another example could be a coastal system where the frequency of grain motion may depend on the frequency of storms and the probability of being deposited on the surface or at depth. Storage times in such a system may last around a year if exposed to storm waves or hundreds to thousands of years if located in backwater deposits. As a final example, a sand grain residing on soil may not move unless disturbed by a biotic process occurring at some frequency (Furbish \& Haff, 2010). The frequency of motion of the sand grain may vary dramatically depending on the local biota and climate with tropical soils potentially having high rates of motion and desert soils potentially having lower rates (Wilkinson et al., 2009), although further evidence is needed.

The effect of storage times on the luminescence of sediment across landscapes has not been directly studied in the context of sediment tracing and fingerprinting. However, we must note that almost all luminescence ages on sedimentary deposits are a measurement of the latest sediment storage time. Recent work on pebbles and cobbles showed that multiple storage and exposure cycles can be detected in the luminescence depthprofiles down the surface of a rock (Freiesleben et al., 2015). Because luminescence is a measure of sediment storage time, luminescence may hold potential for quantifying and answering questions related to sediment storage, sediment connectivity, and preservation potential (Jain et al., 2004).

\subsection{Luminescence Sensitization in Surface Environments}

The luminescence sensitivity is defined as the luminescence intensity per unit dose per unit mass (Gy ${ }^{-1}$ $\cdot \mathrm{kg}^{-1}$ ). Feldspar grains from sediments usually have high luminescence sensitivity compared to quartz grains (Gliganic et al., 2017). The OSL sensitivity is a critical characteristic for sediment dating because low sensitivity hinders the measurement of reliable equivalent doses in quartz (e.g., Preusser et al., 2006). Laboratory experiments indicate that the OSL sensitivity of quartz generally increases after heating (Bøtter-Jensen et al., 1995; Li, 2002; Wintle \& Murray, 1999) or after cycles of light stimulation and irradiation (Bøtter-Jensen et al., 1995; Moska \& Murray, 2006; Pietsch et al., 2008). In nature, the OSL sensitivity of quartz can vary over several orders of magnitude due to its variable temperature conditions of crystallization and long persistence in surface environments as sediment grains (Sawakuchi et al., 2011). Sediments can comprise quartz grains with diverse thermal, irradiation, and bleaching histories, promoting changes in charge traps and recombination centers responsible for luminescence. Various factors potentially control the OSL sensitivity of quartz grains from sediments, including the thermal history of the source rocks (Guralnik, Ankjærgaard Jain, et al., 2015,Guralnik, Jain, et al., 2015; Sawakuchi et al., 2011), wildfires (Rengers et al., 2017), transport as grains in sedimentary systems (Fitzsimmons, 2011; Lü et al., 2014; Pietsch et al., 2008; Zheng et al., 2009), shifts in regional climate (Juyal et al., 2009), and denudation rate of sediment source areas (Sawakuchi et al., 2018).

However, there is a poor understanding of how these factors exactly control quartz OSL sensitivity (Fitzsimmons, 2011). We do know that the OSL sensitivity of quartz can change following illumination and/or regeneration depending on the luminescence signal of interest (Stokes, 1994). Sensitivity changes appear to occur during sediment transport as grains cycle between illumination during transport and regeneration during burial storage (Li \& Wintle, 1992; Pietsch et al., 2008). However, sensitivity changes during these cycles can be hard to predict as sensitivity can either increase or decrease with repeated cycling, although sensitivity generally increases with cycles (Pietsch et al., 2008). In addition, source area geology appears to play a predominant role in controlling OSL sensitivity of quartz from sediments (Fitzsimmons, 2011; Sawakuchi et al., 2018). Sawakuchi et al. (2018) noted in the Amazon River Basin of South America that low sensitivity quartz derives from areas under higher denudation rate, which promote a fast conversion of quartz crystals from parent rocks into sediment grains, whereas high sensitivity quartz occurs in sediments from tectonically stable areas with low denudation rates. This link with source geology at various geographic locations sets the basis for luminescence sensitivity as a provenance tool (Nascimento et al., 2015; Sawakuchi et al., 2012; Sawakuchi et al., 2018; Zular et al., 2015) as discussed in later sections. 


\section{Previous Research on Luminescence as a Sediment Tracer}

There has been a substantial amount of research on luminescence as a sediment tracer; however, much of it has been disparate, focusing on differing geomorphic environments. Here, we attempt to collect and unify each of the major fronts of luminescence sediment tracing. We first describe the basis of the use of luminescence in an environment, then provide an overview of the published literature on this topic, summarize the current state of the science, and then comment on current research needs.

\subsection{Hillslopes and Soils}

Soils and sediments exposed at the Earth's surface are subject to a range of biotic and non-biotic processes that serve to advect and disperse material (Anderson, 2015). Biotic drivers can range from vegetation roots (Roering et al., 2002) to burrowing mammals (Gabet et al., 2003; Winchell et al., 2016) and insects (Kristensen et al., 2015; Rink et al., 2013). Nonbiotic processes range from near surface effects like rain splash (Furbish \& Haff, 2010) to deeper processes such as the growth and collapse of pore space by shrink-swell and freeze-thaw processes or relocation of material by soil forming processes (Anderson, 2015). Understanding the advection and dispersion of sediment grains, here broadly defined as soil mixing, is extremely important for understanding the movement of nutrients and habitats critical for life (Meysman et al., 2006) and for understanding the movement of sediment that sets the pace and form of landscapes on Earth and beyond (Dietrich et al., 2003; Dietrich \& Perron, 2006).

Luminescence has the potential to help answer fundamental questions related to natural soil mixing as its light-sensitive nature preserves information on the paths of grains throughout a soil column (Figure 6). Broadly, particles in soil move via a combination of advective and diffusive motions (Furbish, Roering, Almond, et al., 2018, Furbish, Roering, Keen-Zebert, et al., 2018). These motions are related to the granular dynamics of creep and to external perturbations that alter soil velocity profiles and sediment flux rate (BenDror \& Goren, 2018). The quasi-random motions of sand grains eventually lead to particles reaching the surface (Furbish, Roering, Almond, et al., 2018). When sand or silt grains reach the surface, their luminescence bleaches due to sunlight exposure. If the grain reburies into the soil, the luminescence regenerates by background radiation. The net effect is that the luminescence of a single grain is a function of its surface exposure and burial history, and the average signal of multiple grains at given depth is a function of the relative proportion of surface exposed grains. Individual grains encode information on the time-averaged downward velocity (Heimsath et al., 2002), and the average dose of many grains (e.g., measured using multigrain aliquots) encodes information on the bulk diffusivity of the mixing medium (Furbish, Roering, Keen-Zebert, et al., 2018; Johnson et al., 2014). Both downward mixing velocity and bulk diffusivity can potentially illuminate the various biotic and nonbiotic mixing processes occurring at a given field site and could potentially be used to validate models of soil motions. Coupling a geomorphic process-based model of hillslope particle motions with luminescence (e.g., Furbish, Roering, Keen-Zebert, et al., 2018) produces predictions of the pattern of luminescence at a given field site. The predictions can be tested by measuring the patterns of luminescence and comparing with model results. This is a promising application, as quantifying these processes remains a critical research need.

There is a considerable body of research on the effects of soil mixing on luminescence, and on using luminescence to quantify mixing processes. For the former, soil mixing is often referred to as bioturbation, and there is considerable knowledge on how mixing of buried sediment affects luminescence ages (see Bateman et al., 2003). For the latter, the first apparent applications of luminescence as a soil tracer are by Heimsath et al. (2002). Heimsath et al. (2002) performed a groundbreaking study in which they measured both the soil production rate using cosmogenic beryllium-10 $\left({ }^{10} \mathrm{Be}\right)$ and the single-grain quartz OSL signal for a population of grains. They used their measurements to calibrate a Monte Carlo simulation of particle motions in a creeping soil in the upper Bega Valley in southeastern New South Wales, Australia. Their OSL measurements revealed nonsaturated single grains within the soil. For this to be true, particles in soil must undergo vertical motions, as soil is advected downslope. From their observations, they were able to quantify the downward mixing rate and concluded that mixing was likely independent of depth at their field site. Heimsath et al. (2002) were able to make inferences that provided much needed insight on the nature of soil transport (Wilkinson \& Humphreys, 2005). 

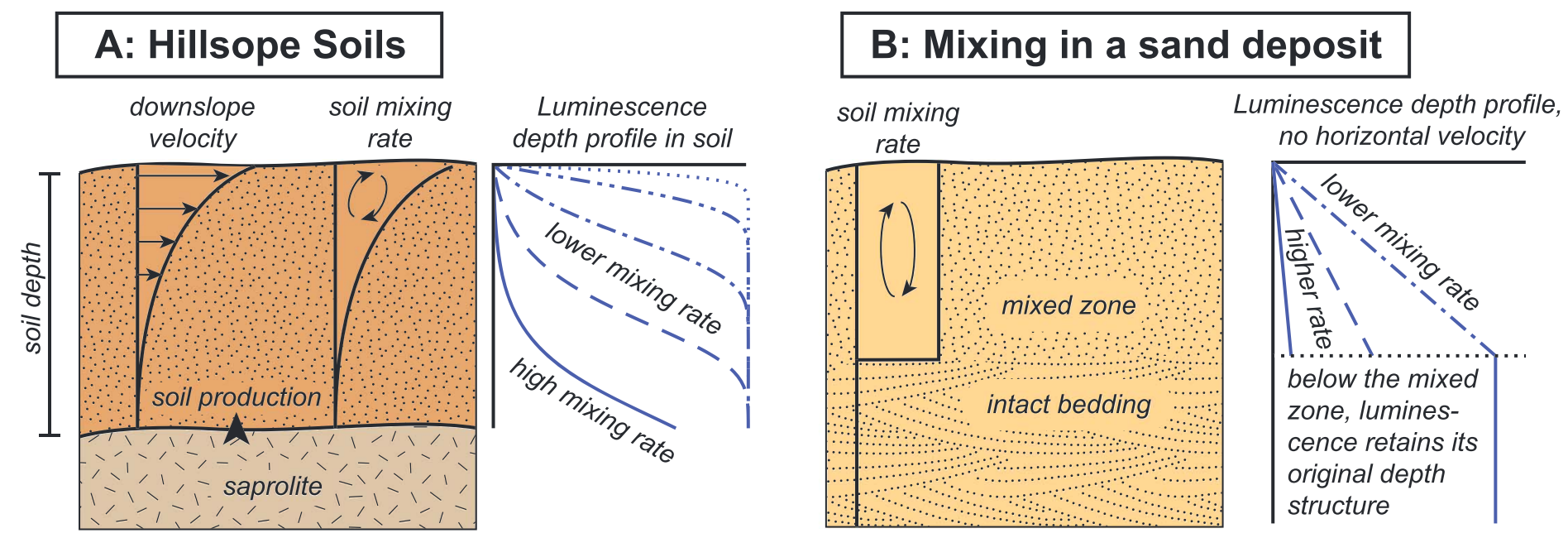

Figure 6. Examples of luminescence depth profiles in mixing soils and sedimentary deposits. Note that the forms of the depth profiles are schematic and will vary based on the specific mixing agents at any given site. (a) Hillslope soils with a component of down-slope driven velocity (Furbish, Roering, Keen-Zebert, et al., 2018). (b) Vertical mixing of a deposit such as a sand sheet (Gliganic et al., 2017).

Stockmann et al. (2013) followed up the work of Heimsath (2002) by measuring the luminescence ages of single grains in soil at Werrikimbe National Park in Australia. In contrast to Heimsath et al. (2002), Stockmann et al. (2013) found evidence of a depth dependence on mixing rate based on their single-grain distributions. Building on this work, Reimann et al. (2017) calculated "soil reworking rates" for a hillslope in northern Spain using the methods of Heimsath et al. (2002) adapted for feldspar IRSL. Reimann et al. (2017) found that their initial soil reworking rates were constant with depth and adapted the technique to factor in depthdependent mixing. The tracer method underwent an important advance by Johnson et al. (2014), who modeled the luminescence of quartz grains in soil using an advection-diffusion equation for a field site in northeastern Queensland, Australia. In their study, Johnson et al. (2014) compared both a linear and exponential depth profile for soil diffusivity and found that the exponential profile best fit the field data, which let them quantify both the diffusivity and the characteristic lengthscale of mixing. Román-Sánchez, Reimann, et al., (2019),Román-Sánchez, Laguna, et al., (2019) merged both the downward velocity and advection-diffusion approaches in their comprehensive study of the Santa Clotilde Critical Zone Observatory in southern Spain. Román-Sánchez, Reimann, et al., (2019), Román-Sánchez, Laguna, et al., (2019) present a large body of work, including analytical solutions to the advection-diffusion equation, a dataset spanning an entire hillslope, and careful analysis of the mixing and transport processes at their field sites. The combined efforts of the above work are important because quantifying the soil mixing/diffusivity and lengthscales of movement opens the door for modeling other processes such as predicting the movement of nutrients and other soil properties.

A concurrent thread in quantifying mixing processes started with the work of Madsen et al. (2011). Madsen et al. (2011) quantified the vertical mixing rates of lugworms in a coastal tidal flat using a novel method similar to the methods used in vertically mixing soils in the section above. Their approach was to conceptualize the mixing of sediment as a process that removed material from below and deposited it on the surface, in effect quantifying this mixing process as an accumulation rate. A numerical model of this process matched field observations. Kristensen et al. (2015) used a similarly novel approach to quantify the soil mixing as a "soil conveyor" driven by termite mount construction in Ghana. Gliganic et al. (2016) used a slightly different method by observing the proportion of light-exposed grains versus depth and by calculating a downward mixing rate based on soil replacement time, an approach like those used in soil science (Richards, 2009; Wilkinson et al., 2009). This is a promising approach because Gliganic et al. (2016) showed a depth dependence on soil mixing and demonstrated how luminescence can directly obtain a useful and specific soil mixing parameter. Recently, Porat et al. (2019) was able to show that one can make inferences on the timescales of soil transport using portable OSL readers. Similarly, Stang et al. (2012) set out to determine whether soil mixing appears in depth profiles of portable luminescence reader measurements of the IRSL and blue stimulated luminescence (BSL) of polymineral aliquots in the San Gabriel Mountains of southern California, USA. 
Stang et al. (2012) compared their luminescence depth profiles with a simple model of uniform grain diffusion and demonstrated that complex mixing profiles must be occurring at this site.

Finally, Furbish, Roering, Almond, et al., (2018), Furbish, Roering, Keen-Zebert, et al., (2018), 2018c), using a Fokker-Planck equation, a specialized probabilistic form of the Master Equation (Fokker, 1914; Planck, 1917), demonstrated how the random paths of particles in a soil lead to changes in the depth profiles of luminescence ages. In, particular, Furbish, Schumer, et al. (2018) demonstrated that particles in soil undergo rarefied transport, meaning that continuum style formulations of advection and diffusion are not applicable in soils a priori. Instead, methods from statistical mechanics, such as a Fokker-Plank approach, are necessary. This advance sets the theoretical basis for connecting measurements of luminescence, particularly single grains, with the probability distributions of particle motions resulting from various soil mixing processes. Importantly, it allows one to estimate a Peclet number (a ratio of advective versus diffusive forces), which is a useful value in studies of soils and landscape evolution.

The current (2019) state of the science of luminescence as a soil mixing tracer is as follows. There are currently three main methods used to infer soil mixing rates from luminescence. The first is to divide the sample's apparent age by the depth to obtain a downward velocity (Heimsath et al., 2002) that can be interpreted in terms of soil mixing as a soil reworking rate (e.g., Reimann et al., 2017). The second is to apply advectiondiffusion style equations on the age versus depth structure of soil (Johnson et al., 2014; Román-Sánchez, Reimann, et al., 2019, Román-Sánchez, Laguna, et al., 2019). The third is to apply a probabilistic model of rarefied sand grains and use the change in single grain luminescence ages versus depth to infer timescales of particle motion and soil Peclet numbers (Furbish, Roering, Almond, et al., 2018, Furbish, Roering, Keen-Zebert, et al., 2018, 2018c). An additional method is to perform a regression of age versus depth to obtain an accumulation rate to track the movement of sediment (e.g., Bruening-Madsen et al., 2017; Kristensen et al., 2015). The choice of method is dependent on the question at hand. For example, the downward velocity method is applicable to some soil mixing parameters (Wilkinson et al., 2009), whereas the advection/diffusion approach may be more similar to other soil mixing tracers such as bomb and cosmogenic isotopes (Kaste et al., 2007). The next steps in advancing this technique lie in testing luminescencebased mixing rates with mixing rates quantified from nonluminescence methods. It will be important to show that one can obtain similar values from different methods. This verification is critical to confirm that luminescence is acting as a tracer. In addition, how specific soil mixing processes alter luminescence depth profiles remains an open question for many processes. These processes could include tree throw, shrinkswell, and biotic burrowing among many others. Using luminescence as a soil mixing tracer provides an important tool to support a wide range of potential future research needs.

\subsection{Fluvial Systems and Landscapes}

While there is a substantial body of literature on understanding bleaching of luminescence in fluvial systems (see bleaching section above), there is substantially less research on directly using luminescence to quantify fluvial transport. Most fluvial-process related research focuses on using chronology of fluvial/alluvial deposits to quantify the rates and timescales of fluvial processes (e.g., Chamberlain et al., 2017; Chamberlain, Törnqvist, et al., 2018; Chamberlain, Wallinga, et al., 2018; Keen-Zebert et al., 2013; Keen-Zebert, 2015; Larkin et al., 2017; Quik \& Wallinga, 2018; Rowland et al., 2005; Tooth et al., 2014; Yanites et al., 2010) or using luminescence, measured in total photon counts in a portable reader (Sanderson \& Murphy, 2010), to infer qualitative changes in fluvial sediment deposition through luminescence depth profiles (MuñozSalinas et al., 2011, 2012, 2013, 2014; Portenga \& Bishop, 2016; Portenga et al., 2016). As dating is beyond the scope of this paper, we will not review the former, but as the latter uses luminescence to provide insight into sediment transport as viewed in an upstream direction, we review it in the "provenance" section further on.

The initial suggestion of the potential of luminescence sediment tracing in rivers stems from a review of luminescence bleaching in fluvial systems by Jain et al. (2004), who observed an inconsistency in the residual doses (due to incomplete bleaching prior to deposition) between the modern samples and those in a stratigraphic context. They related this observation to the preservation potential of the deposited sediment in a given location; the greater the preservation potential, the lesser the chance that the sediment in the stratigraphic record had its luminescence fully zeroed prior to deposition. Thus, they suggested that the degree of luminescence bleaching inferred from the stratigraphic record may be an indicator of the total amount of 


\section{Schematic pattern of luminescence in an idealized river/floodplain system}

\begin{abstract}
All sand grains saturated in

Luminescence

All sand grains fully bleached of luminescence
\end{abstract}

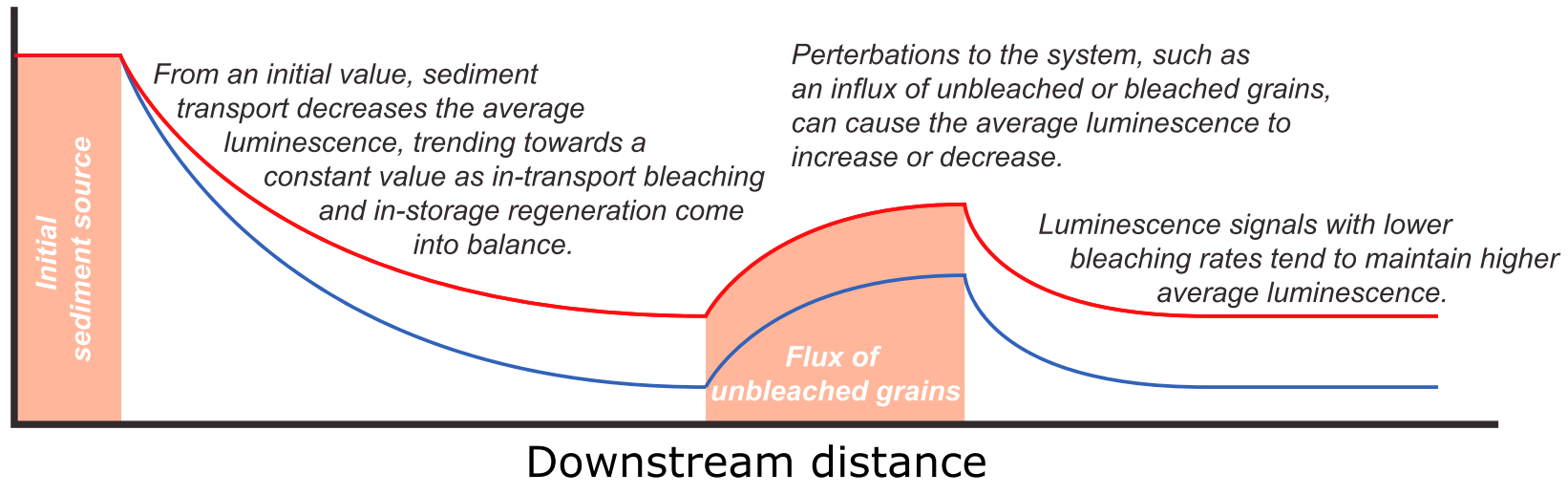

Perterbations to the system, such as an influx of unbleached or bleached grains, luminescence to

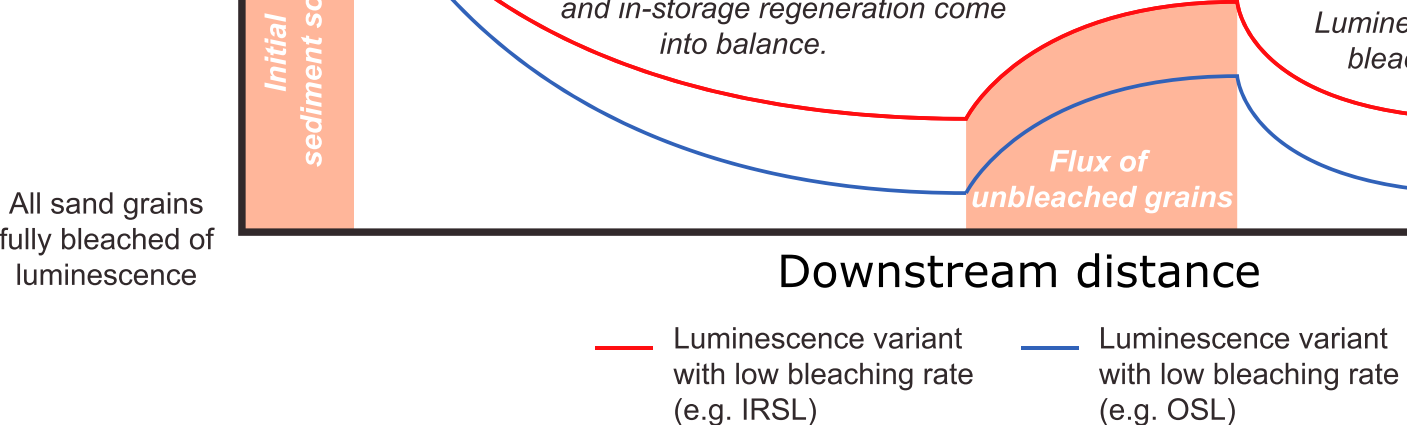
(e.g. IRSL) (e.g. OSL)

Figure 7. Schematic patterns of the luminescence averaged over thousands of grains in an idealized channel/floodplain fluvial system. Adapted from Gray et al. (2017).

transport of grains since detachment from the source. Thus, luminescence potentially contains information on basin wide or smaller scale processes that controlled sediment movement and recycling in the past.

One of the first explicit uses of directly using luminescence to quantify rates of fluvial transport as virtual velocities was performed by McGuire and Rhodes (2015a, 2015b). McGuire and Rhodes (2015a) conceptualized the Mojave River as an idealized channel, wherein sediment sourced from the San Bernardino Mountains undergoes a sediment transport history marked by discrete episodes of rest time between flash flood events. In such a system, the luminescence of sediment undergoes bleaching during transport and regeneration during the inter-flood periods. From this conceptualization, McGuire and Rhodes (2015a) were able to estimate a virtual velocity for fine-grained sediment in the Mojave River. A virtual velocity is a velocity averaged over transport and rest times (Ferguson \& Wathen, 1998; Haschenburger \& Church, 1998; Milan \& Large, 2014) and is useful for evaluating the time needed for signals to propagate through a fluvial system (Pizzuto et al., 2014, 2017). Following on the works of McGuire and Rhodes (2015a, 2015b), Gray et al. (2017) used a combined conservation of mass and conservation of energy approach to develop a theoretical model of luminescence in an idealized channel system involving exchange with a floodplain reservoir (Figure 7). From this theoretical model, one can fit field data and extract estimates for the virtual velocity, characteristic lengthscale of transport, and rates of sediment exchange between channel and floodplain. Gray et al. (2017) expanded the McGuire and Rhodes (2015a, 2015b) concept to a more detailed level of geomorphic processes. However, both McGuire and Rhodes (2015a, 2015b) and Gray et al. (2017) largely simplify bleaching as a process that only occurs during in-channel transport. It is not yet known how to include surface bleaching (Gray \& Mahan, 2015), yet single-grain datasets indicate that both in-channel and surface bleaching is occurring (McGuire \& Rhodes, 2015b). Although these methods require further testing and development, their potential use to quantify sediment transport is a promising new research frontier.

The current state of the science in fluvial luminescence sediment tracing has the hallmarks of a young research field. There are a handful of new methods offering insight into sediment transport, yet many parameters are not fully constrained, and the field has not yet reached a "holy grail" of predicting bulk volumetric sediment fluxes with luminescence. First, McGuire and Rhodes (2015a, 2015b) and Gray et al. $(2017,2018)$ have demonstrated the potential of using luminescence as a downstream tracer in fluvial sediment to quantify virtual velocities. However, important problems remain open for study. For example, we must establish whether luminescence-based transport values match those from other methods and determine why or why not (Gray et al., 2018). Next, we must determine the conditions under which bleaching dominantly occurs in transport or by surface exposure, and the extent to which this is system dependent. Other important problems, such as how various luminescence signals bleach in a subaqueous turbulent flow, include changes in subaqueous wavelengths of light and could be amenable to flume studies using 


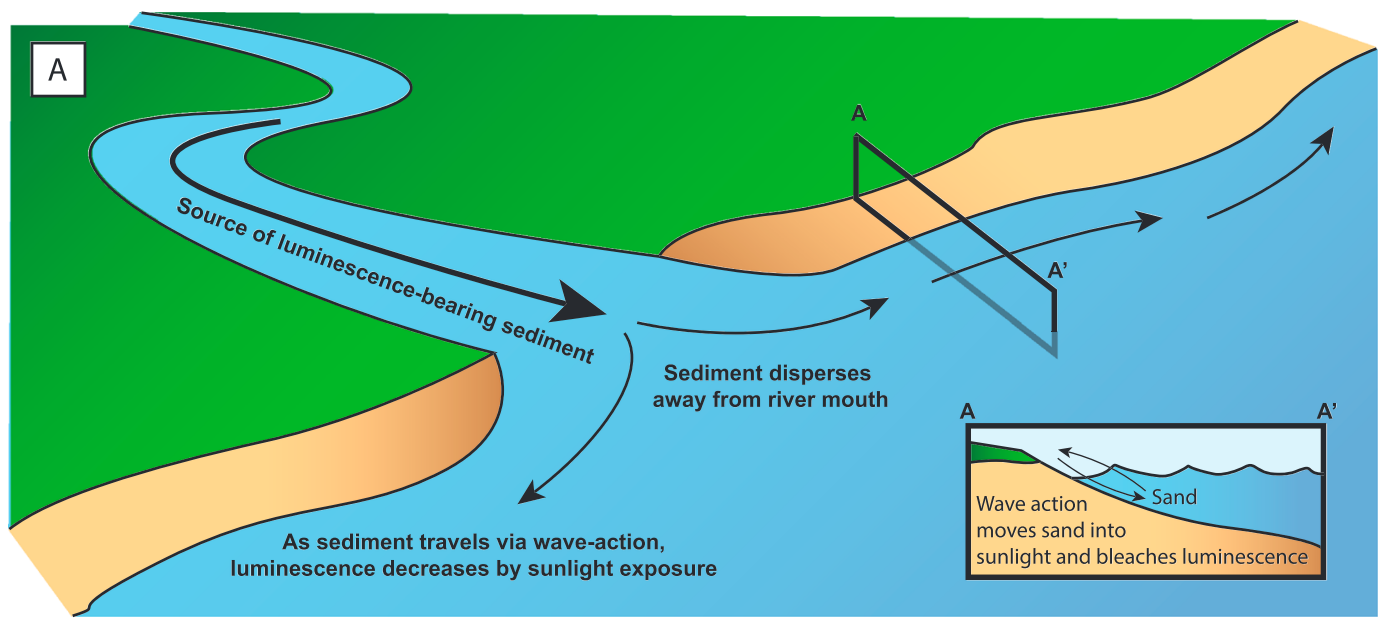

B

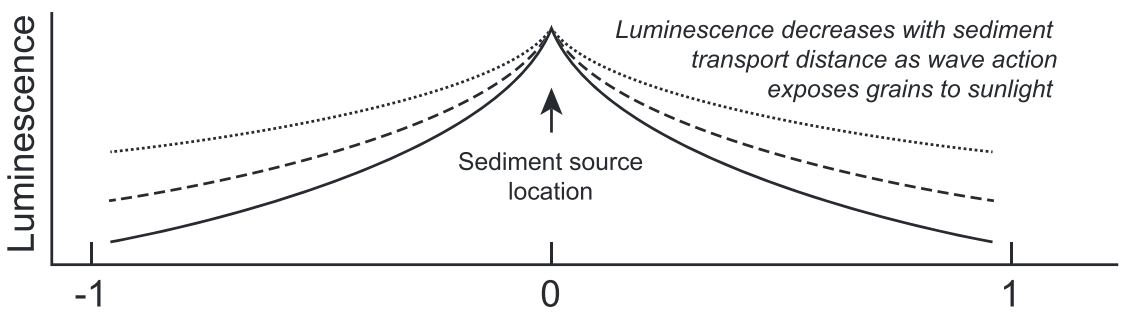

Distance from along coastline from sediment source

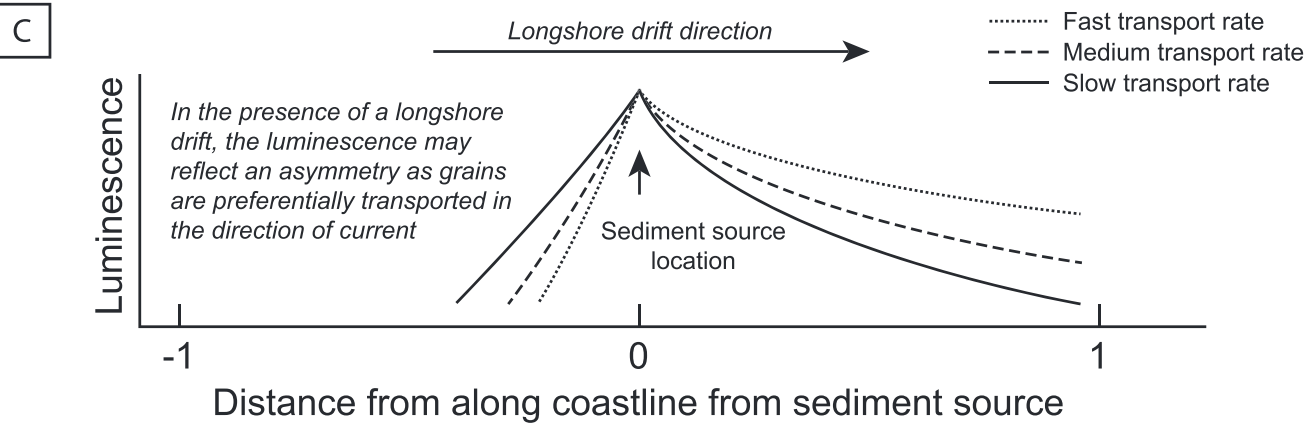

Figure 8. (a) Illustration of luminescence as a coastal sediment tracer and hypothesized bleaching and transport mechanism. (b) Hypothesized tendency of luminescence versus coastline distance from a sediment source, such as a river mouth, at 0. (c) Similar graph to (b) but with a dominant longshore drift direction.

solar simulators. A flume approach could also inform dating efforts by examining controls on beaching rates and depositional patterns. Other problems include understanding how the rate of sediment recycling in a basin affects the residual luminescence in channel sediment (Jain et al., 2004), how the luminescence of channel sediment depends on scale from individual grains and bars up to basins, what the patterns of luminescence are in a meandering floodplain, how mixtures of sediment from various sources appear in channel sediment luminescence, and how grain chemical dissolution, mechanical abrasion, and grain sorting may affect luminescence. Answering these questions may help advance luminescence as a sediment tracer and potentially allow luminescence to be used as a proxy for volumetric sediment fluxes, a key variable in landscape evolution.

\subsection{Coastal Settings}

Luminescence has been used to infer coastal sediment transport (Figure 8) and deposition since the 1990s. One of the earliest applications of luminescence as a sediment tracer appears in Forman (1990), who discovered a correlation between the TL residuals and distance from the outlet of a glacier in a coupled glacialcoastal system. From this finding, Forman (1990) interpreted that TL levels and/or sensitivity may be a potential tool for constraining depositional environments, as grains undergo light exposure during 
sediment transport differently across different systems. The first major follow-up to this research was Rink (1999), who noted that TL intensity in beach deposits were correlated with sample location that tied to prior light exposure during sediment transport. Rink (2003) found that TL signals decrease in magnitude with northerly distance along the Israeli coast. Rink (2003) interpreted this finding as evidence of northward longshore drift as sediment moves from its source in the Nile River delta and receives greater cumulative light exposure progressively reducing the TL signals with further transport. These studies provide some of the first data on changes in luminescence with sediment transport distance, which indicate that luminescence has potential as a sediment tracer in coastal environments.

Later work has greatly expanded the range and methods of luminescence in coastal sediment tracing. Keizars et al. (2008) set out to see if this observed trend of Rink (2003) was present in the St. Joseph Peninsula, Florida. Keizars et al. (2008) found that this trend was present and produced the some of the first quantitative estimates for different grain sizes on average light exposure, residence time, and TL loss rate per unit distance. Liu, Ogawa, et al., (2009), Liu, Kishimoto, et al., (2009), Liu et al., (2011, 2013) presented an important body of research on the Tenryu-Enshunada fluvial/coastal system in Japan. Liu, Ogawa, et al. (2009) observed the coastal pattern of TL measurements and demonstrated a clear signal of elevated TL at the mouth of the Tenryu River that tapers to lower values with distance along the Enshunada coastline. From these data, Liu, Ogawa, et al. (2009) concluded that sediment from the river migrates equally westward and eastward from the river mouth and then estimated the respective long-shore sediment flux. Notably, Liu et al. (2011) demonstrated one of the first practical applications of the TL tracing method in tracking the migration of sand artificially added as erosion control. Liu et al. (2013) give a key synthesis of the work in the region. Reimann et al. (2015) also explored this novel application by measuring the change in luminescence in a sand nourishment site. The work of Reimann et al. (2015) is an important advance for the quantification of sunlight exposure over time and the consideration of the sediment transport and light exposure processes at play. As a final note, Shirai and Hayashizaki (2013), following methods from Shirai et al. (2008), examined the fraction of fully bleached versus unbleached grains in submarine turbidites in a bid to determine a flooding or seismogenic origin for deposits off the coast of Japan. While the percent of bleached grains versus depositional location did not show a clear pattern, Shirai and Hayashizaki (2013) were cleverly able to supplement their findings with grain rounding and make conclusions on sediment sources. These studies represent important advancements on the use of luminescence intensity directly as a coastal sediment tracer.

Of particular note, Ahmed et al. (2013) give a fantastic example of luminescence as a means to quantify coastal sediment transport rates. Ahmed et al. (2013) developed a quantitative model of longshore sediment transport incorporating the sunlight exposure of grains by wave action. Using the decrease in TL as a function of sunlight exposure time, Ahmed et al. (2013) were able to estimate the velocities of sand grains in longshore drift and calculate a volumetric sediment flux, a key parameter in understanding coastal geomorphology. Their work is exciting and an excellent example, as it represents a case where quantitative information can be obtained from luminescence where the resulting values agree with estimates from other methods (Ahmed et al., 2013).

The current state of the science for coastal luminescence sediment tracing is as follows. TL has been observed to correlate with sediment transport distance with sediment that has traveled further appearing more thoroughly bleached than sediment less traveled (Forman, 1990) because of greater light exposure (Rink, 1999). OSL appears to deplete quicker in response to light exposure than TL (Keizars et al., 2008), although this depends on water turbidity and turbulence (Reimann et al., 2015). Over the scale of coastlines, the intensity of natural TL can be observed to decrease with sediment transport distance, which can inform direction of longshore drift (Liu, Ogawa, et al., 2009; Rink, 2003) and potentially the fluxes of sediment (Liu, Kishimoto, et al., 2009; Liu et al., 2011). This was confirmed by Ahmed et al. (2013) who developed a quantitative model for estimating volumetric sediment flux using measurements of TL to infer exposure time. There is notable potential to use these methods to track natural and artificial sediment introduced to control erosion (Liu et al., 2011; Reimann et al., 2015), although some challenges remain because of the random turbulent nature of sediment transport (Reimann et al., 2015). It may be possible to combine the approach of Ahmed et al. (2013) with the multisignal equivalent exposure time methods of Reimann et al. (2015) to provide robust estimates of volumetric sediment flux. There is currently ongoing research activity developing and applying sediment tracing methods in coastal settings (Liu et al., 2011; Liu et al., 2013; Liu \& Sato, 2012; Sato et al., 2011; Sato et al., 2015; Tajima et al., 2011). 
To advance luminescence as a coastal sediment tracer, it is necessary to quantitatively link luminescence signals, such as OSL and TL residuals, with rates of coastal sediment transport. There are some potential ways to accomplish this. An empirical approach comparing measurements of sediment flux with luminescence could provide insight into the quantitative form of the relation. This would be very useful in guiding theoretical efforts. A theoretical modeling approach, coupling luminescence physics of bleaching and regeneration to theoretical models of sediment transport in coastal settings, could give a useful general relation for testing in natural experiments or analog wave flume studies. Possible challenges with this approach would be accurately simulating the transport of sediment under various conditions of wave energy and determining how bleaching of different luminescence signals occurs during surface exposure and/or during wave/tidal transport. Developing luminescence as a quantitative coastal sediment tracer is an important goal with applications to questions of the effects of climate on coastlines and in coastline restoration.

\subsection{Aeolian Transport}

The use of residual luminescence (e.g., equivalent dose) as a sediment tracer in aeolian systems is very unexplored. This may be because subaerial sunlight exposure times in aeolian systems are long enough to fully bleach most luminescence signals (Wintle, 1993), such that little transport information remains stored in the equivalent dose of a sediment grain. However, it may be possible to use luminescence signals that bleach very slowly to perform sediment tracing. Signals with slower bleaching include for example hightemperature post-infrared infrared stimulated luminescence (post-IR IRSL) of potassium feldspar, with signals remaining after several days of light exposure (Kars et al., 2014). Although outside the scope of our review, the use of luminescence dating to quantify landscape change has been very informative on the processes that control aeolian sediment transport in environments ranging from arid (Lancaster, 2008; Singhvi \& Porat, 2008; Stone \& Thomas, 2013) to seasonal wet climates (Ballarini et al., 2003; Murray-Wallace et al., 2002). The use of portable luminescence readers also offers the potential for high-resolution analysis of the sequence of deposition across aeolian features (Stone et al., 2019). There has been notably more work performed in aeolian sediment provenance as discussed further below

\section{Luminescence as a Provenance Tool}

In contrast to sediment tracing, which tracks grain motions often in a downstream direction, sediment fingerprinting observes sediment in an upstream direction to determine sediment sources or provenance. Sediment fingerprinting can be challenging when sediment composition is nondistinct such as when sediment is dominated by quartz and feldspar (Solomon, 1932; Weltje \& von Eynatten, 2004). Fortunately, luminescence characteristics, such as TL or OSL sensitivity, quartz LM-OSL components, and $D_{0}$ values among others, can serve as useful properties to distinguish sediment from different source areas. For example, two sand grains of quartz may be visually nondescript, but manifest very different values of any given luminescence characteristic. In addition, luminescence characteristics do not change the physics of sand grain transport, a facet that is beneficial for understanding natural sediment transport (Reimann et al., 2015). Luminescence characteristics are thought to be a function of geography and source geology as discussed in the previous sections (Jain et al., 2003; Sawakuchi et al., 2018). The following sections describe previous research using luminescence characteristics, especially of quartz and feldspar, to ascertain the sources of sediment in various geomorphic environments.

As a refresher, we discussed how provenance can be divided into geographic provenance methods, which look for specific locations of sediment sources, and geomorphic provenance, which seeks to define geomorphic units or processes (such as landslides) that introduce sediment. The river basin section has the luxury of a significant body of research on provenance, such that we can divide out geographic and geomorphic methods. Admittingly, the lines between these distinctions are often blurred as geomorphic processes can be geographically restricted, so the groupings can be somewhat arbitrary. Other provenance sections have these two merged together.

\subsection{River Basins: Geographic Provenance}

Recently, Haddadchi et al. (2016) and Sawakuchi et al. (2018) opened a new research frontier by applying measurements of the luminescence characteristic sensitivity and LM-OSL components to sediment within river basins (Figure 2). Haddadchi et al. (2016), compared the characteristics of LM-OSL components and 
found that sediment from hillslope soils has significantly different luminescence characteristics than sediment derived from riverbanks. The change is particularly noticeable for the slow component of quartz LM-OSL. Haddadchi et al. (2016) found agreement with radionuclide tracers, which provided strong evidence for the potential of LM-OSL as a provenance tool. This is promising because LM-OSL can be used on quartz sand grains, which is often difficult to fingerprint despite being almost geologically ubiquitous.

Similarly, Sawakuchi et al. (2018) found that distinct regions of the Amazon River Basin appear to produce sediment (silt and sand) with notably different OSL sensitivities, showing a correlation with source area and denudation rate and no correlation with transport distance at the $1000-\mathrm{km}$ scale. In this study, the sensitivity is represented by the relative contribution of the fast component of quartz OSL. The correlation between quartz OSL sensitivity and denudation rate is supported by proxies based on cosmogenic nuclides $\left({ }^{10} \mathrm{Be}\right)$ and elemental ratios. Supporting this finding, Nascimento et al. (2015) demonstrated that the OSL sensitivity of quartz matches with provenance signatures based on heavy mineral analysis. These results indicate that luminescence sensitivity provides an effective sediment provenance tool within the Amazon River Basin, and the results reveal geomorphic controls on OSL sensitivity. Importantly, the relation between denudation rate and OSL sensitivity warrants analysis in other systems to determine whether this correlation relates to changes in geomorphic process induced by higher denudation rates or the correlation derives from source lithology, which is often correlated with denudation rate. In addition, the apparent lack of correlation between sensitivity and downstream distance appears to differ from other research (Gliganic et al., 2017; Pietsch et al., 2008). The differences in sensitivity and LM-OSL components among different rivers could be due to differences in basin size, source lithology, or sediment transport mechanisms (Nian et al., 2018). Answers to these open questions offer would provide further insight into sediment transport mechanics.

The use of luminescence characteristics as a provenance tool in river systems has substantial potential (Haddadchi et al., 2016; Sawakuchi et al., 2018). Important next steps involve repeating these methods in other drainage basins to understand how widely applicable these methods are. The sensitization of quartz OSL in geomorphic systems can be attributed to transport in sedimentary systems due to repeated bleaching-regeneration cycles (e.g., Pietsch et al., 2008) or to source rock weathering, where longer weathering time promotes the increase in OSL sensitivity (Sawakuchi et al., 2018). In both cases, the OSL sensitization is related to the period spent by quartz as grains in Earth's near-surface settings (soils or depositional systems). Thus, another important goal includes understanding the sensitization of quartz grains in terms of solar exposure and near-surface radiation field.

\subsection{River Basins: Geomorphic Provenance}

There is a notable body of work investigating correlations between fluvial depositional patterns and changes in luminescence with depth, with an eye towards inferring geomorphic provenance. The papers in this section largely center around measurements made with a portable luminescence reader (Sanderson \& Murphy, 2010). Portable luminescence readers consist of a photomultiplier tube and light-emitting diode stimulation unit installed above a sample chamber. In contrast to a laboratory-sized luminescence reader, the units do not contain a radiation source. As such, the portable luminescence reader makes measurements of luminescence, as total photon counts emitted from a sample. These total photon counts are expressed as either BSL or IRSL and are usually not normalized for sensitivity (luminescence produced per unit dose). The benefits of these types of measurements are that they are quick, cheap, and can be made in the field at high density relative to standard laboratory readers. The complications are that the data can involve extra variables such as unnormalized inter-sample sensitivity, inclusion of other minerals besides quartz and feldspar, a variable range of grain sizes, and the challenges of ensuring a consistent sample size between aliquots. Despite these complicating factors, measurements of total photon count likely contain information on the age structure and/or partial bleaching of a stratigraphic profile (Sanderson \& Murphy, 2010), which can then be interpreted in terms of geomorphic processes (e.g., Muñoz-Salinas et al., 2011).

Muñoz-Salinas et al. (2011) conducted one of the first studies explicitly using luminescence, as measurements of total photon counts with a portable reader (Sanderson \& Murphy, 2010), as a proxy for changes in fluvial depositional dynamics in three systems. In their sites, Muñoz-Salinas et al. (2011) note correlations between changes in depositional stratigraphy, such as sediment texture and magnetic susceptibility, with the total photon counts, which they tie to changes in fluvial provenance. Their suggested drivers include changes in the entrainment rates of lahar flows, sediment bioturbation, and land-use change following 
the eighteenth century colonization of Australia. Similarly, Muñoz-Salinas et al. (2012) and Muñoz-Salinas et al. (2013) applied this approach to volcano-fluvial lahar deposits and glaciofluvial deposits, respectively, and tied changes in luminescence total photon counts, such as standard deviation, to changes in the upstream sediment transport processes delivering material to the sampled locations. Following up on this work, Muñoz-Salinas et al. (2014) make a strong case for the use of portable luminescence readers as provenance tools in studies of land-use change. The key conclusion from their work is that the upstream soils developed over metasedimentary rocks have low total photon counts and are not likely sources for postcolonization deposits with higher total photon counts as sediment transport downstream should either decrease or keep the total photon counts constant with transport distance. The findings of Muñoz-Salinas et al. (2014) were later shown to be reproducible by data given by Portenga and Bishop (2016), who agreed with interpretations by Muñoz-Salinas et al. (2014). Portenga et al. (2016) would later expand the MuñozSalinas et al. (2014) approach to various other regional sites allowing for a wider-scale interpretation of river basin change. In some systems, interpretation of fluvial sediment provenance can be challenging. For example, Muñoz-Salinas et al. (2016) noted conflicting depth versus total photon counts in deposits from the Usumacinta-Grijalva River Basin, which they interpreted as documenting significant partial bleaching, similarly to many other environments (Muñoz-Salinas, Castillo, \& Arce, 2017).

Provenance interpretations based on portable luminescence reader data can be strengthened when coupled with additional sediment fingerprinting/provenance methods such as meteoric ${ }^{10} \mathrm{Be}$ or ${ }^{137} \mathrm{Cs}$ (MuñozSalinas \& Castillo, 2018). Portenga et al. (2017) presented a new method for using coupled bulk OSL and meteoric ${ }^{10} \mathrm{Be}$ to fingerprint the sources of sediment produced by gully erosion. Gullies are small erosional features that can arise from changes in runoff following changes in vegetation cover (Rengers et al., 2016). Gullies are often considered a geologic hazard, and they can be a primary driver for landscape change and have been associated with human-induced landscape changes (Portenga et al., 2017; Rengers \& Tucker, 2014). Portenga et al. (2017) were able to tie upstream sediment sources with downstream deposits by identifying sediment with high levels of bulk luminescence in the downstream deposits and then locating similarly high levels upstream. Their meteoric ${ }^{10} \mathrm{Be}$ measurements provided further constraints on the likely motions of sediment. Following this correlation, Portenga et al. (2017) inferred that erosion must have occurred to certain scour depths to access the sediment with high bulk luminescence. Further research involving coupled fingerprinting/provenance methods promises to be fruitful as the different mechanics of additional methods can add nuance to interpretations.

Other useful provenance applications involve tying luminescence to landscape-scale variables to infer landscape change. Castillo et al. (2014) observed a slight correlation (coefficient of determination $\left[R^{2}\right]=0.27$ ) in the Jalisco Block of Western Mexico, between IRSL total photon counts and average basin channel steepness index, $K_{s n}$, a normalized parameter subsuming variables such as average rock erodibility within a basin (Wobus et al., 2006). Following up on this observation, Muñoz-Salinas, Castillo, Caballero, et al. (2017) performed a study comparing BSL and IRSL total photon counts with average basin slope and found notable correlations $\left(R^{2}=0.65\right.$ for BSL, $R^{2}=0.78$ for IRSL) for basins along the Oaxaca Fault in Mexico. Basins along the Donaji Fault in Mexico did not show as good a correlation $\left(R^{2}=0.26\right.$ for BSL, $R^{2}=0.12$ for IRSL). However, the Oaxaca dataset demonstrated a negative correlation between mean basin slope and total photon counts. This result is puzzling, as one may expect a higher basin slope to produce higher energy flows and greater delivery of unbleached sediment downstream. Muñoz-Salinas, Castillo, and Arce (2017) suggested that the luminescence versus basin slope relationship is a function of the tectonic motions of the range front faults. Further research is needed to understand how the tectonic motions result in changes in luminescence and if it is via other variable, such as changes in sensitivity, number of luminescence grains, light attenuation through the aliquot, and grain size, are playing a role in these results. Finally, Castillo et al. (2017) found a correlation between BSL/IRSL and Ti/Al ratios in tectonically influenced lakes and suggested that this correlation is controlled by landscape change. These correlations indicate possible connections between luminescence and geomorphic processes that warrant further study.

These observations provide an interesting new dimension to stratigraphic analysis by incorporating burial depth versus bulk luminescence expressed as total photon counts (Sanderson \& Murphy, 2010) and open further questions for future study (Muñoz-Salinas et al., 2013, 2014). In some cases, the roles of changes in luminescence sensitivity and mineral assemblage on total photon counts may need further elucidation before stratigraphic patterns can be converted into fluvial processes. For example, if the total photon 
counts of a unit decrease toward the surface, is this a function of a change in sensitivity, number of luminescent grains in the sample chamber, or local dose rate? These variables could feasibly change the total photon counts of a sample outside of a fluvially-driven change (Bateman et al., 2015; Munyikwa \& Brown, 2014; Sanderson \& Murphy, 2010). It is also possible that a pattern may be present based on the autogenic dynamics of a fluvial system without an externally driven change. For example, the correlation of total photon counts with magnetic susceptibility (Muñoz-Salinas et al., 2011) may also suggest that mineralogies other than quartz and feldspar are playing a role in the results. Is perhaps the relative concentration of other minerals changing due to density sorting during deposition? Finally, Bishop et al. (2010) note a correlation between grain size and total photon counts, which they tie to transport processes, but this observation could feasibly be related to luminescence grain size effects (e.g., TimarGabor et al., 2017). These questions are not to imply that interpretations of changes in fluvial processes based on patterns of total photon counts are unreasonable but that total photon counts involve factors related both to luminescence and fluvial processes. Portenga et al. (2016) carefully analyze the effects of grain size and mineral assemblage on their measurements of total photon counts. This analysis boosts their interpretation that partial bleaching is the primary factor in their measurements and suggests that a similar analysis would benefit similar applications of portable luminescence readers. Other factors that might warrant future exploration are sensitivity recorded in sediment depth profiles, the presence of light-absorbing dark organic matter, and clay coatings on grains.

It is worth noting that the studies above could strongly benefit from an investigation demonstrating exactly how specific fluvial processes result in specific changes in total photon counts. A flume-based study may be ideal for addressing many of these questions, where various fluvial transport parameters can be controlled and the resulting portable reader measurements could be calibrated against variables such as water flow rate and sediment transport rate. Such a study could elucidate how total photon counts should change with fluvial transport, including not just partial bleaching, but also sensitivity and grain size changes.

\subsection{Coastal Sediment Provenance}

The luminescence characteristics of sensitivity (Sawakuchi et al., 2012; Zular et al., 2015) and LM-OSL component ratios (Tsukamoto et al., 2011) are useful as provenance tools for assessing coastal sediment sources (Lü et al., 2016) and can help answer paleoclimatic and other research questions. Sawakuchi et al. (2011, 2012) found that the sensitivity of quartz OSL serves as a useful provenance tool for sediments accumulated in coastal barriers from southeastern Brazil where higher sensitivity indicates longer transport distance, which allows one to distinguish single grains from proximal versus distal sources. From this data, Sawakuchi et al. (2012) inferred temporal changes in storm activity as storms deliver far-traveled sediment to their field site. Storm activity is a key paleoclimatic variable, and the Sawakuchi et al. (2012) study represents a clear example of luminescence sediment tracing to infer the response of coastal sedimentation to Holocene paleoclimate changes. Zular et al. (2015) advanced the Sawakuchi et al. (2012) method by demonstrating how it can be combined with heavy mineral analysis (Zular et al., 2013). Furthermore, Zular et al. (2015) demonstrated that the sensitivity of the $110{ }^{\circ} \mathrm{C}$ TL peak appears better suited than OSL sensitivity for determining provenance. Zular et al. (2015) demonstrated agreement of TL and heavy mineral provenance proxies, which discriminated the contribution of sediments from local and distal sources to build the São Franscisco do Sul coastal barrier in southern Brazil during the Holocene. These works demonstrate the potential of sensitivity to enlighten the workings of coastal transport processes.

The second use of luminescence characteristics in coastal sediment tracing is the use of LM-OSL components to distinguish sediment provenance. Tsukamoto et al. (2011) sought to determine the provenance of sediments in the Japanese coastline hypothesized to consist of a combination of distal aeolian sourced material and sediment from the Japanese mainland. Previous work demonstrated that the ratio of the LM-OSL components differed based on source area (Jain, Botter-Jensen, et al., 2005; Tokuyasu et al., 2010). Tsukamoto et al. (2011) demonstrated that the ratio $F /(F+M)$, where $F$ is the fast component and $M$ is the medium component, can be used to distinguish between grains of different source lithology. This shows that LM-OSL components can serve as a coastal sediment provenance tool.

\subsection{Aeolian Provenance}

A common approach in aeolian luminescence provenance is to examine the sensitivity of quartz grains as a function of source lithology and transport history (Lü \& Sun, 2011; Zheng et al., 2009). For example, Lü et al. 
(2014) found that the luminescence sensitivity in a vertical section of loess changed notably with depth. Lü et al. (2014) then concluded that changes in sensitivity occur as a function of climate-driven shifts in sediment provenance. This is an interesting idea and suggests that further research into the mechanisms of sensitivity change, either by geomorphic transport processes or irradiation during burial, deserves further research.

A parallel approach is to use LM-OSL components and/or characteristic dose $\left(D_{0}\right.$; Table 1) values to trace sediment from source to sink. For example, Gong et al. $(2014,2015)$ demonstrated that sand collected from two different deserts, the Hunshandake and the Taklimakan Deserts, had notably different relative compositions of quartz LM-OSL components and $D_{0}$ values, which they attributed to the differing source lithologies for the desert sands. Tsukamoto et al. (2011) also discovered an aeolian sediment source for their field site using LM-OSL components. Other research into aeolian sediment provenance involves using TL via color images (Ganzawa et al., 1997; Hashimoto et al., 1989; Shimada et al., 2013) and using red TL to fingerprint sediment (Yawata \& Hashimoto, 2004), both of which show promise as tracer methods. As a final note, Munyikwa et al. (2012) were able to use a portable luminescence reader to infer a shift from aeolian sourced to non-aeolian sourced sediments in their field sites.

\section{Conclusions and Future Research Questions}

Luminescence is a dynamic property of mineral sand and silt grains that decays with sunlight exposure and regenerates during burial in sedimentary deposits. The amount of sunlight exposure and average duration of burial a sediment grain experiences during transport depends on its geomorphic environment. As such, the amount of luminescence measured, for example, in a silt or sand grain, or averaged over many grains, encodes information about prior transport history.

There is substantial research and a growing number of studies on the use of luminescence as a sediment tracer and provenance tool. Luminescence has been used to understand sediment transport in rivers and coastal systems, active soils, and used to evaluate model parameters for sediment transport. Future work regarding sediment transport would benefit from a focus on understanding the exact process of light exposure in different geomorphic systems (where and how), the scale dependence on bleaching, and use of this understanding to build/refine location-specific theoretical and computational models of sediment transport. Both single-grain, multigrain, and multisignal approaches are important tools in this direction.

With regard to provenance studies, luminescence characteristics, such as OSL and TL sensitivities and the number of OSL components, can reflect sediment source area characteristics such as lithology and denudation rate. There are demonstrations of the use of luminescence for provenance reconstruction in fluvial, coastal, and aeolian systems. Substantial evidence exists that luminescence sensitivity changes occur during sediment transport after quartz has been detached from the parent rock. Although the exact reasons for this are still not understood, it appears that different catchments may imprint their unique signature that can be useful for differentiating between sediment sources in a stratigraphic record. Previous provenance reconstruction studies have mainly focused on quartz OSL. Feldspar is relatively unexploited but holds important promise because of availability of many different signal types with different physical characteristics making it a tunable luminescence system (Jain \& Ankjærgaard, 2011).

While important work has been done and much research remains, it is evident that luminescence serves as a useful property for sediment tracer applications. In general, previous luminescence tracer studies have mainly focused on quartz and secondarily on feldspar, which are major components of terrigenous sediments. However, quartz and feldspar still have other luminescence signals showing different bleaching and saturation behaviors that can be investigated as sediment tracers and provenance proxies. This includes isothermal TL (Jain, Botter-Jensen, et al., 2005) and thermal transfer OSL (Wang et al., 2006) of quartz and post-infrared IRSL of feldspar (Thomsen et al., 2008). Additionally, sediments have a diverse suite of minerals as minor components that also emit luminescence (Jain et al., 2006; Marfunin, 1979) and whose signals can be tested for provenance analysis. Equally there are many other signals in both quartz and feldspars related to stimulation with higher energies (e.g., violet stimulated luminescence; Jain, 2009) or higher temperature IRSL stimulations (Thomsen et al., 2008) or different luminescence emissions (Jain et al., 2015; Prasad et al., 2017), which may be exploited in a multidimensional luminescence approach to reconstruct sediment transport histories or provenances. 
Acknowledgments

Support for this work was provided by ACS PRF grant: 56754-ND8. Thanks to Dr. Esperanza Muñoz Salinas, Dr. Benjamin Guralnik, and two anonymous reviewers for helpful and constructive reviews.
A clear understanding of luminescence kinetics in combination with theoretical models of sediment movement applied to well constrained field situations will help in the development of powerful luminescence tracers to unravel both current- and palaeo-transport histories. Although we have not discussed pebble-sized sediments (Liu et al., 2019) as this research front is very new, it is becoming clear that rock surface bleaching is a powerful tool that can give direct insights into multiple depositional-erosional events (Freiesleben et al., 2015). Future efforts may warrant a focus on multisignal approach and understanding the physics of luminescence decay or sensitization in a sediment-water-air matrix.

\section{References}

Ahmed, M. T., Sato, S., \& Tajima, Y. (2013). Quantitative estimation of longshore sediment transport based on thermoluminescence: Two case studies around Tenryu and Nile River Mouths. Journal of Coastal Research, 30(3), 537-547.

Aitken, M. J. (1998). Introduction to optical dating: The dating of Quaternary sediments by the use of photon-stimulated luminescence. Oxford, United Kingdom: Clarendon Press.

Alexanderson, H. (2007). Residual OSL signals from modern Greenlandic river sediments. Geochronometria, 26(1), 1-9.

Alexanderson, H., \& Murray, A. S. (2012). Luminescence signals from modern sediments in a glaciated bay, NW Svalbard. Quaternary Geochronology, 10, 250-256.

Anderson, R. S. (2015). Particle trajectories on hillslopes: Implications for particle age and 10Be structure. Journal of Geophysical Research: Earth Surface, 120, 1626-1644. https://doi.org/10.1002/2015JF003479

Anderson, R. S., \& Anderson, S. P. (2010). Geomorphology: The mechanics and chemistry of landscapes. Cambridge: Cambridge University Press.

Arnold, L. J., Bailey, R. M., \& Tucker, G. E. (2007). Statistical treatment of fluvial dose distributions from southern Colorado arroyo deposits. Quaternary Geochronology, 2(1-4), 162-167.

Bailey, R. M., Smith, B. W., \& Rhodes, E. J. (1997). Partial bleaching and the decay form characteristics of quartz OSL. Radiation Measurements, 27(2), 123-136.

Ballarini, M., Wallinga, J., Murray, A. S., van Heteren, S., Oostd, A. P., Bos, A. J. J., \& van Eijk, C. W. E. (2003). Optical dating of young coastal dunes on a decadal time scale. Quaternary Science Reviews, 22, 1011-1017.

Bateman, M. D., Frederick, C. D., Jaiswal, M. K., \& Singhvi, A. K. (2003). Investigations into the potential effects of pedoturbation on luminescence dating. Quaternary Science Reviews, 22(10-13), 1169-1176.

Bateman, M. D., Stein, S., Ashurst, R. A., \& Selby, K. (2015). Instant luminescence chronologies? High resolution luminescence profiles using a portable luminescence reader. Quaternary Geochronology, 30, 141-146.

Bateman, M. D., Swift, D. A., Piotrowski, J. A., Rhodes, E. J., \& Damsgaard, A. (2018). Can glacial shearing of sediment reset the signal used for luminescence dating? Geomorphology, 306, 90-101.

Belmont, P., Willenbring, J. K., Schottler, S. P., Marquard, J., Kumarasamy, K., \& Hemmis, J. M. (2014). Toward generalizable sediment fingerprinting with tracers that are conservative and nonconservative over sediment routing timescales. Journal of Soils and Sediments, 14(8), 1479-1492.

BenDror, E., \& Goren, L. (2018). Controls over sediment flux along soil-mantled hillslopes: Insights from granular dynamics simulations Journal of Geophysical Research: Earth Surface, 123, 924-944. https://doi.org/10.1002/2017JF004351

Berger, G. W. (1990). Effectiveness of natural zeroing of the thermoluminescence in sediments. Journal of Geophysical Research, 95(B8), 12375-12397.

Bishop, P., Munoz-Salinas, E., MacKenzie, A. B., Pulford, I., \& McKibbin, J. (2010). The charcter, volume and implications of sedimenat impounded in mill dams in Scotland: The case of the Baldernock Mill dam in East Dunbartonshire. Earth and Environmental Science Transactions of the Royal Society of Edinburgh, 101(2), 97-110.

Biswas, R. H., Herman, F., King, G. E., \& Braun, J. (2018). Thermoluminescence of feldspar as a multi-thermochronometer to constrain the temporal variation of rock exhumation in the recent past. Earth and Planetary Science Letters, 495, 56-68.

Bøtter-Jensen, L., Agersnap Larsen, N., Mejdahl, V., Poolton, N. R. J., Morris, M. F., \& McKeever, S. W. S. (1995). Luminescence sensitivity changes in quartz as a result of annealing. Radiation Measurements, 24, 535-541.

Bøtter-Jensen, L., McKeever, S. W., \& Wintle, A. G. (2003). Optically stimulated luminescence dosimetry. Amsterdam, Netherlands: Elsevier. https://doi.org/10.1016/B978-044450684-9/50091-X

Bradley, D. N. (2017). Direct observation of heavy-tailed storage times of bed load tracer particles causing anomalous superdiffusion. Geophysical Research Letters, 44, 12,227-12,235. https://doi.org/10.1002/2017GL075045

Bradley, D. N., Tucker, G. E., \& Benson, D. A. (2010). Fractional dispersion in a sand bed river. Journal of Geophysical Research, 115, F00A09. https://doi.org/10.1029/2009jf001268

Bradley, D. N., \& Tucker, G. E. (2012). Measuring gravel transport and dispersion in a mountain river using passive radio tracers. Earth Surface Processes and Landforms, 37(10), 1034-1045.

Bradley, D. N., \& Tucker, G. E. (2013). The storage time, age, and erosion hazard of laterally accreted sediment on the floodplain of a simulated meandering river. Journal of Geophysical Research: Earth Surface, 118, 1308-1319. https://doi.org/10.1002/jgrf.20083

Breuning-Madsen, H., Kristensen, J. Å., Awadzi, T. W., \& Murray, A. S. (2017). Early cultivation and bioturbation cause high long-term soil erosion rates in tropical forests: OSL based evidence from Ghana. Catena, 151, 130-136.

Brown, N. D., Rhodes, E. J., \& Harrison, T. M. (2017). Using thermoluminescence signals from feldspars for low-temperature thermochronology. Quaternary Geochronology, 42, 31-41.

Bulur, E. (1996). An alternative technique for optically stimulated luminescence (OSL) experiment. Radiation Measurements, 26(5), 701-709.

Burbank, D. W., \& Anderson, R. S. (2009). Tectonic geomorphology. Malden, MA: John Wiley \& Sons.

Buylaert, J. P., Thiel, C., Murray, A. S., Vandenberghe, D. A., Yi, S., \& Lu, H. (2011). IRSL and post-IR IRSL residual doses recorded in modern dust samples from the Chinese Loess Plateau. Geochronometria, 38(4), 432

Castillo, M., Ferrari, L., \& Muñoz-Salinas, E. (2017). Knickpoint retreat and landscape evolution of the Amatlán de Cañas half-graben (northern sector of Jalisco Block, western Mexico). Journal of South American Earth Sciences, 77, 108-122. 
Castillo, M., Muñoz-Salinas, E., \& Ferrari, L. (2014). Response of a landscape to tectonics using channel steepness indices (ksn) and OSL: A case of study from the Jalisco Block, Western Mexico. Geomorphology, 221, 204-214.

Chamberlain, E., \& Wallinga, J. (2018). Fluvial sediment pathways enlightened by OSL bleaching of river sediments and deltaic deposits. Earth Surface Dynamics Discussion. https://doi.org/10.5194/esurf-2018-76

Chamberlain, E. L., Törnqvist, T. E., Shen, Z., Mauz, B., \& Wallinga, J. (2018). Anatomy of Mississippi Delta growth and its implications for coastal restoration. Science Advances, 4(4), eaar4740.

Chamberlain, E. L., Wallinga, J., Reimann, T., Goodbred, S. L. Jr., Steckler, M. S., Shen, Z., \& Sincavage, R. (2017). Luminescence dating of delta sediments: Novel approaches explored for the Ganges-Brahmaputra-Meghna Delta. Quaternary Geochronology, 41, 97-111.

Chamberlain, E. L., Wallinga, J., \& Shen, Z. (2018). Luminescence age modeling of variably-bleached sediment: Model selection and input. Radiation Measurements. https://doi.org/10.1016/j.radmeas.2018.06.007

Chen, R., \& Pagonis, V. (2011). Thermally and optically stimulated luminescence: a simulation approach. Chichester, United Kingdom: John Wiley \& Sons.

Choi, J. H., Duller, G. A. T., Wintle, A. G., \& Cheong, C.-S. (2006). Luminescence character-istics of quartz from the Southern Kenyan Rift Valley: dose estimation using LM-OSL SAR. Radiation Measurements, 41, 847-854.

Collins, A. L., Pulley, S., Foster, I. D., Gellis, A., Porto, P., \& Horowitz, A. J. (2017). Sediment source fingerprinting as an aid to catchment management: A review of the current state of knowledge and a methodological decision-tree for end-users. Journal of Environmental Management, 194, 86-108.

Cunningham, A. C., Evans, M., \& Knight, J. (2015). Quantifying bleaching for zero-age fluvial sediment: A Bayesian approach. Radiation Measurements, 81, 55-61.

Cunningham, A. C., Wallinga, J., Hobo, N., Versendaal, A. J., Makaske, B., \& Middelkoop, H. (2015). Re-evaluating luminescence buria doses and bleaching of fluvial deposits using Bayesian computational statistics. Earth Surface Dynamics, 3(1), 55.

Davis, C. M., \& Fox, J. F. (2009). Sediment fingerprinting: Review of the method and future improvements for allocating nonpoint source pollution. Journal of Environmental Engineering, 135(7), 490-504.

Dietrich, W. E., Bellugi, D. G., Sklar, L. S., Stock, J. D., Heimsath, A. M., \& Roering, J. J. (2003). Geomorphic transport laws for predicting landscape form and dynamics. Geophysical Monograph-American Geophysical Union, 135, 103-132.

Dietrich, W. E., \& Perron, J. T. (2006). The search for a topographic signature of life. Nature, 439(7075), 411.

Ditlefsen, C. (1992). Bleaching of K-feldspars in turbid water suspensions: A comparison of photo-and thermoluminescence signals. Quaternary Science Reviews, 11(1-2), 33-38.

Duller, G. A. (2008). Single-grain optical dating of Quaternary sediments: Why aliquot size matters in luminescence dating. Boreas, 37(4), 589-612.

Duller, G. A. T. (2012). Improving the accuracy and precision of equivalent doses determined using the optically stimulated luminescence signal from single grains of quartz. Radiation Measurements, 47(9), 770-777.

Einstein, H. A. (1937). Bedload transport as a probability problem. Sedimentation, 1027, C1-C105.

Ferguson, R. I., \& Wathen, S. J. (1998). Tracer-pebble movement along a concave river profile: Virtual velocity in relation to grain size and shear stress. Water Resources Research, 34(8), 2031-2038.

Fiebig, M., \& Preusser, F. (2007). Investigating the amount of zeroing in modern sediments of River Danube, Austria. Quaternary Geochronology, 2(1-4), 143-149.

Fitzsimmons, K. (2011). An assessment of the luminescence sensitivity of Australian quartz with respect to sediment history. Geochronometria, 38(3), 199-208.

Fokker, A. D. (1914). Die mittlere Energie rotierender elektrischer Dipole im Strahlungsfeld. Annalen der Physik, 348(5), 810-820.

Forman, S. L. (1990). Thermoluminescence properties of fiord sediments from Engelskbukta, western Spitsbergen, Svalbard: A new tool for deciphering depositional environment? Sedimentology, 37(2), 377-384.

Freiesleben, T., Sohbati, R., Murray, A., Jain, M., Al Khasawneh, S., Hvidt, S., \& Jakobsen, B. (2015). Mathematical model quantifies multiple daylight exposure and burial events for rock surfaces using luminescence dating. Radiation Measurements, 81, 16-22.

Fruergaard, M., Pejrup, M., Murray, A. S., \& Andersen, T. J. (2015). On luminescence bleaching of tidal channel sediments. Geografisk Tidsskrift-Danish Journal of Geography, 115(1), 57-65.

Fuchs, M., \& Lang, A. (2009). Luminescence dating of hillslope deposits-A review. Geomorphology, 109(1-2), 17-26.

Fuchs, M., Straub, J., \& Zöller, L. (2005). Residual luminescence signals of recent river flood sediments: A comparison between quartz and feldspar of fine-and coarse-grain sediments. Ancient TL, 23(1), 25-30.

Furbish, D. J., Fathel, S. L., Schmeeckle, M. W., Jerolmack, D. J., \& Schumer, R. (2017). The elements and richness of particle diffusion during sediment transport at small timescales. Earth Surface Processes and Landforms, 42(1), 214-237.

Furbish, D. J., \& Haff, P. K. (2010). From divots to swales: Hillslope sediment transport across divers length scales. Journal of Geophysical Research, 115, F03001. https://doi.org/10.1029/2009JF001576

Furbish, D. J., Roering, J. J., Almond, P., \& Doane, T. H. (2018). Soil particle transport and mixing near a hillslope crest: 1. Particle ages and residence times. Journal of Geophysical Research: Earth Surface, 123, 1052-1077. https://doi.org/10.1029/2017JF004315

Furbish, D. J., Roering, J. J., Keen-Zebert, A., Almond, P., Doane, T. H., \& Schumer, R. (2018). Soil particle transport and mixing near a hillslope crest: 2. Cosmogenic nuclide and optically stimulated luminescence tracers. Journal of Geophysical Research: Earth Surface, 123, 1078-1093. https://doi.org/10.1029/2017JF004316

Furbish, D. J., Schumer, R., \& Keen-Zebert, A. (2018). The rarefied (non-continuum) conditions of tracer particle transport in soils, with implications for assessing the intensity and depth dependence of mixing from geochronology. Earth Surface Dynamics, 6(4), 1169-1202.

Gabet, E. J., Reichman, O. J., \& Seabloom, E. W. (2003). The effects of bioturbation on soil processes and sediment transport. Annual Review of Earth and Planetary Sciences, 31(1), 249-273.

Ganzawa, Y., Watanabe, Y., Osanai, F., \& Hashimoto, T. (1997). TL color images from quartzes of loess and tephra in China and Japan. Radiation Measurements, 27(2), 383-388.

Gellis, A. C., Fuller, C. C., van Metre, P., Filstrup, C. T., Tomer, M. D., Cole, K. J., \& Sabitov, T. Y. (2018). Combining sediment fingerprinting with age-dating sediment using fallout radionuclides for an agricultural stream, Walnut Creek, Iowa, USA. Journal of Soils and Sediments, 1-23.

Gellis, A. C., \& Walling, D. E. (2011). Sediment source fingerprinting (tracing) and sediment budgets as tools in targeting river and watershed restoration programs. In A. Simon, S. J. Bennett, \& J. M. Castro (Eds.), Stream restoration in dynamic fluvial systems: scientific approaches, analyses, and tools, Geophys. Monogr. Ser., (Vol. 194, pp. 263-291). Washington, D. C: American Geophysical Union.

Gemmell, A. M. (1988). Zeroing of the TL signal in sediment undergoing fluvioglacial transport. An example from Austerdalen, western Norway. Quaternary Science Reviews, 7, 339-345. 
Gemmell, A. M. (1994a). Thermoluminescence in suspended sediment of glacier meltwater streams. Journal of Glaciology, 40(134), 158-166

Gemmell, A. M. (1994b). Environmental controls on the TL age of modern (zero-age) proglacial outwash sediments. Quaternary Geochronology (Quaternary Science Reviews), 13, 485-489.

Gemmell, A. M. (1997). Fluctuations in the thermoluminescence signal of suspended sediment in an alpine glacial meltwater stream. Quaternary Science Reviews (Quaternary Geochronology), 16, 281-290.

Gemmell, A. M. (1999). IRSL from fine-grained glacifluvial sediment. Quaternary Geochronology, 18, $207-215$.

Gemmell, A. M. D. (1985). Zeroing of the TL signal of sediment undergoing fluvial transportation: A laboratory experiment. Nuclear Tracks and Radiation Measurements (1982), 10(4-6), 695-702.

Gliganic, L. A., Cohen, T. J., Meyer, M., \& Molenaar, A. (2017). Variations in luminescence properties of quartz and feldspar from modern fluvial sediments in three rivers. Quaternary Geochronology, 41, 70-82.

Gliganic, L. A., Cohen, T. J., Slack, M., \& Feathers, J. K. (2016). Sediment mixing in aeolian sandsheets identified and quantified using single-grain optically stimulated luminescence. Quaternary Geochronology, 32, 53-66.

Gong, Z., Sun, J., \& Lü, T. (2015). Investigating the components of the optically stimulated luminescence signals of quartz grains from sand dunes in China. Quaternary Geochronology, 29, 48-57.

Gong, Z., Sun, J., Lü, T., \& Tian, Z. (2014). Investigating the optically stimulated luminescence dose saturation behavior for quartz grains from dune sands in China. Quaternary Geochronology, 22, 137-143.

Govin, A., Holzwarth, U., Heslop, D., Keeling, L. F., Zabel, M., Mulitza, S., et al. (2012). Distribution of major elements in Atlantic surface sediments (36oN-49oS): Imprint of terrigenous input and continental weathering. Geochemistry, Geophysics, Geosystems, 13, Q01013. https://doi.org/10.1029/2011GC003785

Gray, H. J., \& Mahan, S. A. (2015). Variables and potential models for the bleaching of luminescence signals in fluvial environments. Quaternary International, 362, 42-49.

Gray, H. J., Tucker, G. E., \& Mahan, S. A. (2018). Application of a luminescence-based sediment transport model. Geophysical Research Letters, 45, 6071-6080. https://doi.org/10.1029/2018GL078210

Gray, H. J., Tucker, G. E., Mahan, S. A., McGuire, C., \& Rhodes, E. J. (2017). On extracting sediment transport information from measurements of luminescence in river sediment. Journal of Geophysical Research: Earth Surface, 122, 654-677. https://doi.org/10.1002/ 2016JF003858

Guralnik, B., Ankjærgaard Jain, M., Murray, A. S., Müller, A., Wälle, M., Lowick, S. E., et al. (2015). OSL-thermochronometry using bedrock quartz: A note of caution. Quaternary Geochronology, 25, 37-48.

Guralnik, B., Jain, M., Herman, F., Ankjærgaard, C., Murray, A. S., Valla, P. G., et al. (2015). OSL-thermochronometry of feldspar from the KTB borehole, Germany. Earth and Planetary Science Letters, 423, 232-243. https://doi.org/10.1016/j.epsl.2015.04.032

Guralnik, B., Li, B., Jain, M., Chen, R., Paris, R. B., Murray, A. S., et al. (2015). Radiation-induced growth and isothermal decay of infraredstimulated luminescence from feldspar. Radiation Measurements, 81, 224-231. https://doi.org/10.1016/j.radmeas.2015.02.011

Guralnik, B., \& Sohbati, R. (2019). Fundamentals of luminescence Photo-and thermochronometry. Advances In Physics And Applications Of Optically And Thermally Stimulated Luminescence, 399. https://doi.org/10.1142/9781786345790_0011

Guzmán, G., Quinton, J. N., Nearing, M. A., Mabit, L., \& Gómez, J. A. (2013). Sediment tracers in water erosion studies: Current approaches and challenges. Journal of Soils and Sediments, 13(4), 816-833.

Haddadchi, A., Olley, J., \& Pietsch, T. (2016). Using LM-OSL of quartz to distinguish sediments derived from surface-soil and channel erosion. Hydrological Processes, 30(4), 637-647.

Haddadchi, A., Ryder, D. S., Evrard, O., \& Olley, J. (2013). Sediment fingerprinting in fluvial systems: review of tracers, sediment sources and mixing models. International Journal of Sediment Research, 28(4), 560-578.

Haschenburger, J. K., \& Church, M. (1998). Bed material transport estimated from the virtual velocity of sediment. Earth Surface Processes and Landforms: The Journal of the British Geomorphological Group, 23(9), 791-808.

Hashimoto, T., Yokosaka, K., Habuki, H., \& Hayashi, Y. (1989). Provenance search of dune sands using thermoluminescence colour images (TLCIs) from quartz grains. International Journal of Radiation Applications and Instrumentation. Part D. Nuclear Tracks and Radiation Measurements, 16(1), 3-10.

Hassan, F. A. (1978). Sediments in archaeology: Methods and implications for palaeoenvironmental and cultural analysis. Journal of Field Archaeology, 5(2), 197-213.

Hassan, M. A., \& Ergenzinger, P. (2003). Use of tracers in fluvial geomorphology. Tools in Fluvial Geomorphology, $397-423$.

Heimsath, A. M., Chappell, J., Spooner, N. A., \& Questiaux, D. G. (2002). Creeping soil. Geology, 30(2), 111-114.

Heimsath, A. M., \& Ehlers, T. A. (2005). Quantifying rates and timescales of geomorphic processes. Earth Surface Processes and Landforms, 30(8), 917-921.

Herman, F., Rhodes, E. J., Braun, J., \& Heiniger, L. (2010). Uniform erosion rates and relief amplitude during glacial cycles in the Southern Alps of New Zealand, as revealed from OSL-thermochronology. Earth and Planetary Science Letters, 297(1-2), 183-189.

Hoffmann, T. (2015). Sediment residence time and connectivity in non-equilibrium and transient geomorphic systems. Earth-Science Reviews, 150, 609-627.

Hooke, J. (2003). Coarse sediment connectivity in river channel systems: a conceptual framework and methodology. Geomorphology, 56(1-2), 79-94.

Hu, G., Zhang, J. F., Qiu, W. L., \& Zhou, L. P. (2010). Residual OSL signals in modern fluvial sediments from the Yellow River (HuangHe) and the implications for dating young sediments. Quaternary Geochronology, 5(2-3), 187-193.

Huntley, D. J. (2006). An explanation of the power-law decay of luminescence. Journal of Physics: Condensed Matter, $18(4), 1359$.

Huntley, D. J., Godfrey-Smith, D. I., \& Thewalt, M. L. (1985). Optical dating of sediments. Nature, 313(5998), 105.

Jacobs, Z. (2008). Luminescence chronologies for coastal and marine sediments. Boreas, 37(4), 508-535.

Jain, M. (2009). Extending the dose range: Probing deep traps in quartz with $3.06 \mathrm{eV}$ photons. Radiation Measurements, 44(5-6), 445-452.

Jain, M., Andersen, C. E., Bøtter-Jensen, L., Murray, A. S., Haack, H., \& Bridges, J. C. (2006). Luminescence dating on Mars: OSL characteristics of Martian analogue materials and GCR dosimetry. Radiation Measurements, 41(7-8), 755-761.

Jain, M., \& Ankjærgaard, C. (2011). Towards a non-fading signal in feldspar: Insight into charge transport and tunnelling from timeresolved optically stimulated luminescence. Radiation Measurements, 46(3), 292-309.

Jain, M., Bøtter-Jensen, L., Murray, A. S., Denby, P. M., Tsukamoto, S., \& Gibling, M. R. (2005). Revisiting TL: Dose measurement beyond the OSL range using SAR. Ancient TL, 23, 9-24.

Jain, M., Guralnik, B., \& Andersen, M. T. (2012). Stimulated luminescence emission from localized recombination in randomly distributed defects. Journal of Physics: Condensed Matter, 24(38), 385402. 
Jain, M., Murray, A. S., \& Bøtter-Jensen, L. (2003). Characterisation of blue-light stimulated luminescence components in different quartz samples: implications for dose measurement. Radiation Measurements, 37(4-5), 441-449.

Jain, M., Murray, A. S., \& Bøtter-Jensen, L. (2004). Optically stimulated luminescence dating: How significant is incomplete light exposure in fluvial environments? Quaternaire, 15(1), 143-157.

Jain, M., Murray, A. S., Bøtter-Jensen, L., \& Wintle, A. G. (2005). A single-aliquot regenerative-dose method based on IR (1.49 eV) bleaching of the fast OSL component in quartz. Radiation Measurements, 39(3), 309-318.

Jain, M., Sohbati, R., Guralnik, B., Murray, A. S., Kook, M., Lapp, T., et al. (2015). Kinetics of infrared stimulated luminescence from feldspars. Radiation Measurements, 81, 242-250. https://doi.org/10.1016/j.radmeas.2015.02.006

Jaiswal, M., Chen, Y., Kale, V., \& Achyuthan, H. (2009). Residual luminescence in quartz from slack water deposits in Kaveri Basin, South India: A single aliquot approach. Geochronometria, 33(1), 1-8.

Jeong, G. Y., \& Choi, J. H. (2012). Variations in quartz OSL components with lithology, weathering and transportation. Quaternary Geochronology, 10, 320-326.

Johnson, M. O., Mudd, S. M., Pillans, B., Spooner, N. A., Keith Fifield, L., Kirkby, M. J., \& Gloor, M. (2014). Quantifying the rate and depth dependence of bioturbation based on optically-stimulated luminescence (OSL) dates and meteoric 10Be. Earth Surface Processes and Landforms, 39(9), 1188-1196.

Just, J., Dekkers, M. J., von Dobeneck, T., van Hoesel, A., \& Bickert, T. (2012). Signatures and significance of aeolian, fluvial, bacterial and diagenetic magnetic mineral fractions in Late Quaternary marine sediments off Gambia, NW Africa. Geochemistry, Geophysics, Geosystems, 13, Q0AO02. https://doi.org/10.1029/2012GC004146

Juyal, N., Pant, R. K., Basavaiah, N., Bhushan, R., Jain, M., Saini, N. K., et al. (2009). Reconstruction of Last Glacial to early Holocene monsoon variability from relict lake sediments of the Higher Central Himalaya, Uttrakhand, India. Journal of Asian Earth Sciences, 34(3), 437-449. https://doi.org/10.1016/j.jseaes.2008.07.007

Kars, R. H., Reimann, T., Ankjærgaard, C., \& Wallinga, J. (2014). Bleaching of the post-IR IRSL signal: New insights for feldspar luminescence dating. Boreas, 43(4), 780-791.

Kaste, J. M., Heimsath, A. M., \& Bostick, B. C. (2007). Short-term soil mixing quantified with fallout radionuclides. Geology, 35(3), 243-246. Keen-Zebert, A. (2015). Luminescence, fluvial sediments. In W. Rink, \& J. Thompson (Eds.), Encyclopedia of Scientific Dating Methods, (pp. 465-469). Dordrecht: Springer.

Keen-Zebert, A., Tooth, S., Rodnight, H., Duller, G. A. T., Roberts, H. M., \& Grenfell, M. (2013). Late Quaternary floodplain reworking and the preservation of alluvial sedimentary archives in unconfined and confined river valleys in the eastern interior of South Africa. Geomorphology, 185, 54-66.

Keizars, K. Z., Forrest, B. M., \& Rink, W. J. (2008). Natural residual thermoluminescence as a method of analysis of sand transport along the coast of the St. Joseph Peninsula, Florida. Journal of Coastal Research, 242, 500-507. https://doi.org/10.2112/04-0406.1

King, G. E., Guralnik, B., Valla, P. G., \& Herman, F. (2016). Trapped-charge thermochronometry and thermometry: A status review. Chemical Geology, 446, 3-17.

King, G. E., Robinson, R. A. J., \& Finch, A. A. (2013). Apparent OSL ages of modern deposits from Fåbergstølsdalen, Norway: Implications for sampling glacial sediments. Journal of Quaternary Science, 28(7), 673-682.

King, G. E., Robinson, R. A. J., \& Finch, A. A. (2014). Towards successful OSL sampling strategies in glacial environments: Deciphering the influence of depositional processes on bleaching of modern glacial sediments from Jostedalen, Southern Norway. Quaternary Science Reviews, 89, 94-107.

King, G. E., Sanderson, D. C., Robinson, R. A., \& Finch, A. A. (2014). Understanding processes of sediment bleaching in glacial settings using a portable OSL reader. Boreas, 43(4), 955-972.

Knight, J., \& Evans, M. (2018). Luminescence dating, sediment analysis, and flood dynamics on the Sabie River, South Africa. Geomorphology, 319, 1-14.

Koiter, A. J., Owens, P. N., Petticrew, E. L., \& Lobb, D. A. (2013). The behavioural characteristics of sediment properties and their implications for sediment fingerprinting as an approach for identifying sediment sources in river basins. Earth-Science Reviews, 125, 24-42.

Kristensen, J. A., Thomsen, K. J., Murray, A. S., Buylaert, J. P., Jain, M., \& Breuning-Madsen, H. (2015). Quantification of termite bioturbation in a savannah ecosystem: Application of OSL dating. Quaternary Geochronology, 30, 334-341.

Kuhns, C. K., Larsen, N. A., \& McKeever, S. W. S. (2000). Characteristics of LM-OSL from several different types of quartz. Radiation Measurements, 32(5-6), 413-418.

Laceby, J. P., Evrard, O., Smith, H. G., Blake, W. H., Olley, J. M., Minella, J. P., \& Owens, P. N. (2017). The challenges and opportunities of addressing particle size effects in sediment source fingerprinting: A review. Earth-Science Reviews, 169, 85-103.

Lancaster, N. (2008). Desert dune dynamics and development: Insights from luminescence dating. Boreas, 37(4), 559-573.

Larkin, Z. T., Tooth, S., Ralph, T. J., Duller, G. A., McCarthy, T., Keen-Zebert, A., \& Humphries, M. S. (2017). Timescales, mechanisms, and controls of incisional avulsions in floodplain wetlands: Insights from the Tshwane River, semiarid South Africa. Geomorphology, 283, 158-172.

Lauer, J., \& Willenbring, J. (2010). Steady state reach-scale theory for radioactive tracer concentration in a simple channel/floodplain system. Journal of Geophysical Research, 115, F04018. https://doi.org/10.1029/2009JF001480

Leopold, L. B. (1966). Channel and hillslope processes in a semiarid area, New Mexico, (Vol. 352). Washington D. C: US Government Printing Office.

Li, F., Pan, B., Lai, Z., Gao, H., \& Ou, X. (2018). Identifying the degree of luminescence signal bleaching in fluvial sediments from the Inner Mongolian reaches of the Yellow River. Geochronometria, 45(1), 82-96.

Li, S. H. (2002). Luminescence sensitivity changes of quartz by bleaching, annealing and UV exposure. Radiation Effects and Defects in Solids, 157(3), 357-364

Li, S. H., \& Wintle, A. G. (1992). Luminescence sensitivity change due to bleaching of sediments. International Journal of Radiation Applications and Instrumentation. Part D. Nuclear Tracks and Radiation Measurements, 20(4), 567-573.

Lindvall, A., Stjern, R., \& Alexanderson, H. (2017). Bleaching of quartz OSL signals under natural and laboratory light conditions. Ancient $T L, 35(2), 12-20$

Liu, H., Hamamoto, A., \& Sato, S. (2011). Monitoring the nourished sand longshore movement based on feldspar luminescence measurement. In The Proceedings of the Coastal Sediments 2011: In 3 Volumes (pp. 57-70)

Liu, H., Kishimoto, S., Takagawa, T., Shirai, M., \& Sato, S. (2009). Investigation of the sediment movement along the Tenryu-Enshunada fluvial system based on feldspar thermoluminescence properties. Journal of Coastal Research, 255, 1096-1105. https://doi.org/10.2112/ 08-1065.1 
Liu, H., Ogawa, Y., Takagawa, T., \& Sato, S. (2009). Longshore sediment movement along the Enshunada coast inferred from feldspar thermoluminescence. In Proceedings Of Coastal Dynamics 2009: Impacts of Human Activities on Dynamic Coastal Processes (With CD ROM) (pp. 1-12)

Liu, H., \& Sato, S. (2012). Evaluation on the sediment movement along the Miyazaki coast in terms of feldspar luminescence features. In Asian And Pacific Coasts 2011 (Pp. 1525-1532).

Liu, H., Takagawa, T., \& Sato, S. (2013). Sand transport and sedimentary features based on feldspar thermoluminescence: A synthesis of the Tenryu-Enshunada fluvial system, Japan. Journal of Coastal Research, 30(1), 120-129.

Liu, J., Cui, F., Murray, A. S., Sohbati, R., Jain, M., Gao, H., et al. (2019). Resetting of the luminescence signal in modern riverbed cobbles along the course of the Shiyang River, China. Quaternary Geochronology, 49, 184-190. https://doi.org/10.1016/j.quageo.2018.04.004

Liu, J., Cui, F., Murray, A. S., Sohbati, R., Jain, M., Gao, H., et al. (2018). Resetting of the luminescence signal in modern riverbed cobbles along the course of the Shiyang River, China. Quaternary Geochronology, 49, 184-190.

López, G. I., Goodman-Tchernov, B. N., \& Porat, N. (2018). OSL over-dispersion: A pilot study for the characterisation of extreme events in the shallow marine realm. Sedimentary Geology, 378, 35-51. https://doi.org/10.1016/j.sedgeo.2018.09.002

Lowick, S. E., Preusser, F., \& Wintle, A. G. (2010). Investigating quartz optically stimulated luminescence dose-response curves at high doses. Radiation Measurements, 45(9), 975-984.

Lü, T., \& Sun, J. (2011). Luminescence sensitivities of quartz grains from eolian deposits in northern China and their implications for provenance. Quaternary Research, 76(2), 181-189.

Lü, T., Sun, J., Li, S. H., Gong, Z., \& Xue, L. (2014). Vertical variations of luminescence sensitivity of quartz grains from loess/paleosol of Luochuan section in the central Chinese Loess Plateau since the last interglacial. Quaternary Geochronology, 22, $107-115$.

Lü, T. Y., Gong, Z. J., \& Xue, L. (2016). Research on provenance using luminescence sensitivity of quartz grains: Progress and prospect. Geological Bulletin of China, 35(8), 1324-1331.

Mabit, L., Benmansour, M., Abril, J. M., Walling, D. E., Meusburger, K., Iurian, A. R., et al. (2014). Fallout 210Pb as a soil and sediment tracer in catchment sediment budget investigations: A review. Earth-Science Reviews, 138, 335-351. https://doi.org/10.1016/j. earscirev.2014.06.007

Madsen, A. T., \& Murray, A. S. (2009). Optically stimulated luminescence dating of young sediments: A review. Geomorphology, 109(1-2), 3-16.

Madsen, A. T., Murray, A. S., Jain, M., Andersen, T. J., \& Pejrup, M. (2011). A new method for measuring bioturbation rates in sandy tidal flat sediments based on luminescence dating. Estuarine, Coastal and Shelf Science, 92(3), 464-471.

Marfunin, A. S. (1979). Physics of minerals and inorganic materials: an introduction. Springer.

McKeever, S. W. S., \& Chen, R. (1997). Luminescence models. Radiation Measurements, 27(5-6), 625-661.

McGuire, C., \& Rhodes, E. J. (2015a). Determining fluvial sediment virtual velocity on the Mojave River using K-feldspar IRSL: Initial assessment. Quaternary International, 362, 124-131.

McGuire, C., \& Rhodes, E. J. (2015b). Downstream MET-IRSL single-grain distributions in the Mojave River, southern California: Testing assumptions of a virtual velocity model. Quaternary Geochronology, 30, 239-244.

Meade, R. H. (2007). Transcontinental moving and storage: The Orinoco and Amazon Rivers transfer the Andes to the Atlantic. In A. Gupta (Ed.), Large Rivers: Geomorphology and Management, (pp. 45-64). Chichester, U. K.: John Wiley and Sons.

Meysman, F. J., Middelburg, J. J., \& Heip, C. H. (2006). Bioturbation: A fresh look at Darwin's last idea. Trends in Ecology \& Evolution, 21(12), 688-695.

Milan, D. J., \& Large, A. R. (2014). Magnetic tracing of fine-sediment over pool-riffle morphology. Catena, 115, 134-149.

Morton, A. C. (1985). Heavy minerals in provenance studies. In Provenance of arenites, (pp. 249-277). Dordrecht: Springer.

Moska, P., \& Murray, A. S. (2006). Stability of the quartz fast-component in insensitive samples. Radiation Measurements, 41, 878-885.

Mukundan, R., Walling, D. E., Gellis, A. C., Slattery, M. C., \& Radcliffe, D. E. (2012). Sediment source fingerprinting: Transforming from a research tool to a management tool. JAWRA Journal of the American Water Resources Association, 48(6), $1241-1257$.

Mulitza, S., Heslop, D., Pittauerova, D., Fischer, H. W., Meyer, I., Stuut, J.-B. W., et al. (2010). Increase in African dust flux at the onset of commercial agriculture in the Sahel region. Nature, 466, 226-228. https://doi.org/10.1038/nature09213

Muñoz-Salinas, E., Bishop, P., Sanderson, D., \& Kinnaird, T. (2014). Using OSL to assess hypotheses related to the impacts of land use change with the early nineteenth century arrival of Europeans in south-eastern Australia: An exploratory case study from Grabben Gullen Creek, New South Wales. Earth Surface Processes and Landforms, 39(12), 1576-1586.

Muñoz-Salinas, E., Bishop, P., Sanderson, D. C., \& Zamorano, J. J. (2011). Interpreting luminescence data from a portable OSL reader: Three case studies in fluvial settings. Earth Surface Processes and Landforms, 36(5), 651-660.

Muñoz-Salinas, E., Bishop, P., Zamorano, J. J., \& Sanderson, D. (2012). Sedimentological processes in lahars: Insights from optically stimulated luminescence analysis. Geomorphology, 136(1), 106-113.

Muñoz-Salinas, E., \& Castillo, M. (2018). Assessing conservation practices in Amalacaxco Gorge (Izta-Popo National Park, Central Mexico) using fallout 137 CS and Optically Stimulated Luminescence (OSL). Journal of Mountain Science, 15(3), 447-460.

Muñoz-Salinas, E., Castillo, M., \& Arce, J. L. (2017). OSL signal resetting in young deposits determined with a pulsed photon-stimulated luminescence (PPSL) unit. Boreas, 46(2), 325-337.

Muñoz-Salinas, E., Castillo, M., Caballero, L., \& Lacan, P. (2017). Understanding landscape dynamics of the Sierra de Juarez, southern Mexico: An exploratory approach using inherited luminescence signals. Journal of South American Earth Sciences, 76, $208-217$.

Muñoz-Salinas, E., Castillo, M., Sanderson, D., \& Kinnaird, T. (2013). Unraveling paraglacial activity on Sierra de Gredos, Central Spain: A study based on geomorphic markers, stratigraphy and OSL. Catena, 110, 207-214.

Muñoz-Salinas, E., Castillo, M., Sanderson, D., Kinnaird, T., \& Cruz-Zaragoza, E. (2016). Using three different approaches of OSL for the study of young fluvial sediments at the coastal plain of the Usumacinta-Grijalva River Basin, southern Mexico. Earth Surface Processes and Landforms, 41(6), 823-834.

Munyikwa, K., \& Brown, S. (2014). Rapid equivalent dose estimation for eolian dune sands using a portable OSL reader and polymineralic standardised luminescence growth curves: Expedited sample screening for OSL dating. Quaternary Geochronology, 22, 116-125.

Munyikwa, K., Brown, S., \& Kitabwalla, Z. (2012). Delineating stratigraphic breaks at the bases of postglacial eolian dunes in centra Alberta, Canada using a portable OSL reader. Earth Surface Processes and Landforms, 37(15), 1603-1614.

Murray, A. S., Olley, J. M., \& Caitcheon, G. G. (1995). Measurement of equivalent doses in quartz from contemporary water-lain sediments using optically stimulated luminescence. Quaternary Science Reviews, 14(4), 365-371.

Murray, A. S., Thomsen, K. J., Masuda, N., Buylaert, J. P., \& Jain, M. (2012). Identifying well-bleached quartz using the different bleaching rates of quartz and feldspar luminescence signals. Radiation Measurements, 47(9), 688-695. 
Murray-Wallace, C. V., Banerjee, D., Bourman, R. P., Olley, J. M., \& Brooke, B. P. (2002). Optically stimulated luminescence dating of Holocene relict foredunes, Guinchen Bay, South Australia. Quaternary Science Reviews, 21(8-9), 1077-1086.

Nascimento, D. R. Jr., Sawakuchi, A. O., Guedes, C. C., Giannini, P. C., Grohmann, C. H., \& Ferreira, M. P. (2015). Provenance of sands from the confluence of the Amazon and Madeira rivers based on detrital heavy minerals and luminescence of quartz and feldspar. Sedimentary Geology, 316, 1-12.

Nian, X., Zhang, W., Qiu, F., Qin, J., Wang, Z., Sun, Q., et al. (2018). Luminescence characteristics of quartz from Holocene delta deposits of the Yangtze River and their provenance implications. Quaternary Geochronology, 49, 131-137.

Olley, J., Caitcheon, G., \& Murray, A. (1998). The distribution of apparent dose as determined by optically stimulated luminescence in small aliquots of fluvial quartz: Implications for dating young sediments. Quaternary Science Reviews, 17(11), 1033-1040.

Pietsch, T. J., Olley, J. M., \& Nanson, G. C. (2008). Fluvial transport as a natural luminescence sensitiser of quartz. Quaternary Geochronology, 3(4), 365-376.

Pizzuto, J., Keeler, J., Skalak, K., \& Karwan, D. (2017). Storage filters upland suspended sediment signals delivered from watersheds. Geology, 45(2), 151-154.

Pizzuto, J., Schenk, E. R., Hupp, C. R., Gellis, A., Noe, G., Williamson, E., et al. (2014). Characteristic length scales and time-averaged transport velocities of suspended sediment in the mid-Atlantic Region, USA. Water Resources Research, 50, 790-805. https://doi.org/ 10.1002/2013WR014485

Planck, M. (1917). Über einen Satz der statistischen Dynamik und seine Erweiterung in der Quantentheorie. Reimer.

Porat, N., López, G. I., Lensky, N., Elinson, R., Avni, Y., Elgart-Sharon, Y., et al. (2019). Using portable OSL reader to obtain a time scale for soil accumulation and erosion in archaeological terraces, the Judean Highlands, Israel. Quaternary Geochronology, 49, 65-70. https:// doi.org/10.1016/j.quageo.2018.04.001

Porat, N., Zilberman, E., Amit, R., \& Enzel, Y. (2001). Residual ages of modern sediments in an hyperarid region, Israel. Quaternary Science Reviews, 20(5-9), 795-798.

Portenga, E. W., \& Bishop, P. (2016). Confirming geomorphological interpretations based on portable OSL reader data. Earth Surface Processes and Landforms, 41(3), 427-432.

Portenga, E. W., Bishop, P., Gore, D. B., \& Westaway, K. E. (2016). Landscape preservation under post-European settlement alluvium in the south-eastern Australian tablelands, inferred from portable OSL reader data. Earth Surface Processes and Landforms, 41(12), 1697-1707

Portenga, E. W., Bishop, P., Rood, D. H., \& Bierman, P. R. (2017). Combining bulk sediment OSL and meteoric 10Be fingerprinting techniques to identify gully initiation sites and erosion depths. Journal of Geophysical Research: Earth Surface, 122, 513-527. https://doi.org/ 10.1002/2016JF004052

Prasad, A. K., Poolton, N. R., Kook, M., \& Jain, M. (2017). Optical dating in a new light: A direct, non-destructive probe of trapped electrons Scientific Reports, 7(1), 12097.

Prescott, J. R., \& Hutton, J. T. (1994). Cosmic ray contributions to dose rates for luminescence and ESR dating: Large depths and long-term time variations. Radiation Measurements, 23(2-3), 497-500.

Preusser, F., Ramseyer, K., \& Schlüchter, C. (2006). Characterisation of low OSL intensity quartz from the New Zealand Alps. Radiation Measurements, 41(7-8), 871-877.

Przegiętka, K., Molewski, P., Juśkiewicz, W., Palczewski, P., \& Chabowski, M. (2016). Characterisation and OSL dating of modern fluvial sediments in the lower Vistula River: Testing the zeroing assumption. Bulletin of Geography. Physical Geography Series, 10(1), 107-121.

Quik, C., \& Wallinga, J. (2018). Reconstructing lateral migration rates in meandering systems-a novel Bayesian approach combining optically stimulated luminescence (OSL) dating and historical maps. Earth Surface Dynamics, 6(3), 705-721.

Reimann, T., Notenboom, P. D., De Schipper, M. A., \& Wallinga, J. (2015). Testing for sufficient signal resetting during sediment transport using a polymineral multiple-signal luminescence approach. Quaternary Geochronology, 25, 26-36.

Reimann, T., Román-Sánchez, A., Vanwalleghem, T., \& Wallinga, J. (2017). Getting a grip on soil reworking-Single-grain feldspar luminescence as a novel tool to quantify soil reworking rates. Quaternary Geochronology, 42, 1-14.

Rendell, H. M., Webster, S. E., \& Sheffer, N. L. (1994). Underwater bleaching of signals from sediment grains: New experimental data. Quaternary Science Reviews, 13(5-7), 433-435.

Rengers, F. K., Pagonis, V., \& Mahan, S. A. (2017). Can thermoluminescence be used to determine soil heating from a wildfire? Radiation Measurements, 107, 119-127.

Rengers, F. K., \& Tucker, G. E. (2014). Analysis and modeling of gully headcut dynamics, North American high plains. Journal of Geophysical Research: Earth Surface, 119, 983-1003. https://doi.org/10.1002/2013JF002962

Rengers, F. K., Tucker, G. E., \& Mahan, S. A. (2016). Episodic bedrock erosion by gully-head migration, Colorado High Plains, USA. Earth Surface Processes and Landforms, 41(11), 1574-1582.

Rhodes, E. J. (2011). Optically stimulated luminescence dating of sediments over the past 200,000 years. Annual Review of Earth and Planetary Sciences, 39, 461-488.

Richards, P. J. (2009). Quantification of bioturbation and its impacts on soil and slope processes (Doctoral dissertation, Macquarie University).

Richardson, C. A. (2001). Residual luminescence signals in modern coastal sediments. Quaternary Science Reviews, 20(5-9), 887-892.

Rink, W. J. (1999). Quartz luminescence as a light-sensitive indicator of sediment transport in coastal processes. Journal of Coastal Research, 148-154.

Rink, W. J. (2003). Thermoluminescence of quartz and feldspar sand grains as a tracer of nearshore environmental processes in the southeastern Mediterranean Sea. Journal of Coastal Research, 19, 723-730.

Rink, W. J., Dunbar, J. S., Tschinkel, W. R., Kwapich, C., Repp, A., Stanton, W., \& Thulman, D. K. (2013). Subterranean transport and deposition of quartz by ants in sandy sites relevant to age overestimation in optical luminescence dating. Journal of Archaeological Science, 40(4), 2217-2226.

Ritchie, J. C., \& McHenry, J. R. (1990). Application of radioactive fallout cesium-137 for measuring soil erosion and sediment accumulation rates and patterns: a review. Journal of Environmental Quality, 19(2), 215-233.

Rittenour, T. M. (2008). Luminescence dating of fluvial deposits: applications to geomorphic, palaeoseismic and archaeological research Boreas, 37(4), 613-635.

Roering, J. J., Almond, P., Tonkin, P., \& McKean, J. (2002). Soil transport driven by biological processes over millennial time scales. Geology, 30(12), 1115-1118

Roering, J. J., Kirchner, J. W., \& Dietrich, W. E. (1999). Evidence for nonlinear, diffusive sediment transport on hillslopes and implications for landscape morphology. Water Resources Research, 35(3), 853-870. 
Román-Sánchez, A., Laguna, A., Reimann, T., Giráldez, J. V., Peña, A., \& Vanwalleghem, T. (2019). Bioturbation and erosion rates along the soil-hillslope conveyor belt, part 2: quantification using an analytical solution of the diffusion-advection equation. Earth Surface Processes and Landforms, 44, 2066-2080.

Román-Sánchez, A., Reimann, T., Wallinga, J., \& Vanwalleghem, T. (2019). Bioturbation and erosion rates along the soil-hillslope conveyor belt. Part 1: Insights from single-grain feldspar luminescence. Earth Surface Processes and Landforms. https://doi.org/10.1002/ esp.4628

Rowland, J. C., Lepper, K., Dietrich, W. E., Wilson, C. J., \& Sheldon, R. (2005). Tie channel sedimentation rates, oxbow formation age and channel migration rate from optically stimulated luminescence (OSL) analysis of floodplain deposits. Earth Surface Processes and Landforms, 30(9), 1161-1179.

Sanderson, D. C., Bishop, P., Stark, M., Alexander, S., \& Penny, D. (2007). Luminescence dating of canal sediments from Angkor Borei, Mekong Delta, southern Cambodia. Quaternary Geochronology, 2(1-4), 322-329.

Sanderson, D. C., \& Murphy, S. (2010). Using simple portable OSL measurements and laboratory characterisation to help understand complex and heterogeneous sediment sequences for luminescence dating. Quaternary Geochronology, 5(2-3), 299-305.

Sato, S., Kishimoto, S., \& Hiramatsu, H. (2011). Long-term evolution of sand and gravel beaches on the Miyazaki Coast. Coastal Engineering Proceedings, 1(32), 57.

Sato, S., Nishiguchi, K., \& Yamanaka, Y. (2015). Tsunami sediment analysis based on luminescence measurement. In The Proceedings Of The Coastal Sediments 2015.

Sawakuchi, A. O., Blair, M. W., DeWitt, R., Faleiros, F. M., Hyppolito, T., \& Guedes, C. C. F. (2011). Thermal history versus sedimentary history: OSL sensitivity of quartz grains extracted from rocks and sediments. Quaternary Geochronology, 6(2), 261-272.

Sawakuchi, A. O., Guedes, C. C. F., DeWitt, R., Giannini, P. C. F., Blair, M. W., Nascimento, D. R., \& Faleiros, F. M. (2012). Quartz OSL sensitivity as a proxy for storm activity on the southern Brazilian coast during the Late Holocene. Quaternary Geochronology, 13, 92-102.

Sawakuchi, A. O., Jain, M., Mineli, T. D., Nogueira, L., Bertassoli, D. J., Häggi, C., et al. (2018). Luminescence of quartz and feldspar fingerprints provenance and correlates with the source area denudation in the Amazon River basin. Earth and Planetary Science Letters, 492, 152-162. https://doi.org/10.1016/j.epsl.2018.04.006

Schielein, P., \& Lomax, J. (2013). The effect of fluvial environments on sediment bleaching and Holocene luminescence ages-A case study from the German Alpine Foreland. Geochronometria, 40(4), 283-293.

Shimada, A., Takada, M., \& Toyoda, S. (2013). Characteristics of ESR signals and TLCLs of quartz included in various source rocks and sediments in Japan: A clue to sediment provenance. Geochronometria, 40(4), 334-340.

Shirai, M., \& Hayashizaki, R. (2013). Transport process of sand grains from fluvial to deep marine regions estimated by luminescence of feldspar: Example from the K umano area, central J apan. Island Arc, 22(2), 242-257.

Shirai, M., Tsukamoto, S., \& Kondo, R. (2008). Transport-depositional processes of present fluvial deposits estimated from OSL intensity of sand-sized grains. The Quaternary Research, 47(6), 377-389.

Singarayer, J. S., \& Bailey, R. M. (2003). Further investigations of the quartz optically stimulated luminescence components using linear modulation. Radiation Measurements, 37(4-5), 451-458.

Singarayer, J. S., Bailey, R. M., Ward, S., \& Stokes, S. (2005). Assessing the completeness of optical resetting of quartz OSL in the natural environment. Radiation Measurements, 40(1), 13-25.

Singhvi, A. K., \& Porat, N. (2008). Impact of luminescence dating on geomorphological and palaeoclimate research in drylands. Boreas, 37(4), 536-558.

Sohbati, R., Liu, J., Jain, M., Murray, A., Egholm, D., Paris, R., \& Guralnik, B. (2018). Centennial-to millennial-scale hard rock erosion rates deduced from luminescence-depth profiles. Earth and Planetary Science Letters, 493, 218-230.

Sohbati, R., Murray, A. S., Chapot, M. S., Jain, M., \& Pederson, J. (2012). Optically stimulated luminescence (OSL) as a chronometer for surface exposure dating. Journal of Geophysical Research, 117, B09202. https://doi.org/10.1029/2012jb009383

Solomon, J. D. (1932). The heavy mineral assemblages of the Great chalky boulder-clay and Cannon-shot gravels of East Anglia, and their significance. Geological Magazine, 69, 314-320.

Spencer, J. Q., \& Sanderson, D. C. (1994). Mapping thermal exposure by luminescence thermometry. Radiation Measurements, 23(2-3), 465-468.

Stang, D. M., Rhodes, E. J., \& Heimsath, A. M. (2012). Assessing soil mixing processes and rates using a portable OSL-IRSL reader: preliminary determinations. Quaternary Geochronology, 10, 314-319.

Stockmann, U., Minasny, B., Pietsch, T. J., \& McBratney, A. B. (2013). Quantifying processes of pedogenesis using optically stimulated luminescence. European Journal of Soil Science, 64(1), 145-160.

Stokes, S. (1994). The timing of OSL sensitivity changes in a natural quartz. Radiation Measurements, 23(2-3), 601-605.

Stokes, S., Bray, H. E., \& Blum, M. D. (2001). Optical resetting in large drainage basins: tests of zeroing assumptions using single-aliquot procedures. Quaternary Science Reviews, 20(5-9), 879-885.

Stone, A., Bateman, M. D., Burrough, S. L., Garzanti, E., Limonta, M., Radeff, G., \& Telfer, M. W. (2019). Using a portable luminescence reader for rapid age assessment of aeolian sediments for reconstructing dunefield landscape evolution in southern Africa. Quaternary Geochronology, 49, 57-64.

Stone, A. E. C., \& Thomas, D. S. G. (2013). Casting new light on late Quaternary environmental and palaeohydrological change in the Namib Desert: A review of the application of optically stimulated luminescence in the region. Journal of Arid Environments, 93, 40-58.

Syvitski, J. P., Vörösmarty, C. J., Kettner, A. J., \& Green, P. (2005). Impact of humans on the flux of terrestrial sediment to the global coastal ocean. Science, 308(5720), 376-380.

Tajima, Y., Mochizuki, S., Funatake, S., Bandula, W., Lal, S., Sobue, S. I., \& Sato, S. (2011). Investigation of nearshore sedimentary characteristics along the west coast of Sri Lanka based on the analysis of satellite images and feldspar thermoluminescence. Journal of Japan Society of Civil Engineers, Ser. B2 (Coastal Engineering), 67(2), I_631-I_635.

Takayama, S. (1965). Bedload movement in torrential mountain streams. Tokyo Geography Papers, 9, 169-188.

Thomsen, K. J., Murray, A. S., Jain, M., \& Bøtter-Jensen, L. (2008). Laboratory fading rates of various luminescence signals from feldsparrich sediment extracts. Radiation Measurements, 43, 1474-1486.

Timar-Gabor, A., Buylaert, J. P., Guralnik, B., Trandafir-Antohi, O., Constantin, D., Anechitei-Deacu, V., et al. (2017). On the importance of grain size in luminescence dating using quartz. Radiation Measurements, 106, 464-471. https://doi.org/10.1016/j.radmeas.2017.01.009

Tokuyasu, K., Tanaka, K., Tsukamoto, S., \& Murray, A. (2010). The characteristics of OSL signal from quartz grains extracted from modern sediments in Japan. Geochronometria, 37(1), 13-19.

Tooth, S., McCarthy, T., Rodnight, H., Keen-Zebert, A., Rowberry, M., \& Brandt, D. (2014). Late Holocene development of a major fluvia discontinuity in floodplain wetlands of the Blood River, eastern South Africa. Geomorphology, 205, 128-141. 
Tóth, O., Sipos, G., Kiss, T., \& Bartyik, T. (2017). Variation of OSL residual doses in terms of coarse and fine grain modern sediments along the Hungarian section of the Danube. Geochronometria, 44(1), 319-330.

Truelsen, J. L., \& Wallinga, J. A. K. O. B. (2003). Zeroing of the OSL signal as a function of grain size: investigating bleaching and thermal transfer for a young fluvial sample. Geochronometria, 22(1), e8.

Tsukamoto, S., Nagashima, K., Murray, A. S., \& Tada, R. (2011). Variations in OSL components from quartz from Japan sea sediments and the possibility of reconstructing provenance. Quaternary International, 234(1-2), 182-189.

Tucker, G. E., \& Hancock, G. R. (2010). Modelling landscape evolution. Earth Surface Processes and Landforms, 35(1), 28-50.

Vandenberghe, D., Derese, C., \& Houbrechts, G. (2007). Residual doses in recent alluvial sediments from the Ardenne (S Belgium). Geochronometria, 28(1), 1-8.

Viers, J., Roddaz, M., Filizola, N., Guyot, J. L., Sondag, F., Brunet, P., et al. (2008). Seasonal and provenance controls on NdeSr isotopic compositions of Amazon rivers suspended sediments and implications for Nd and Sr fluxes exported to the Atlantic Ocean. Earth and Planetary Science Letters, 274(3), 511-523.

Walling, D. E. (2013). The evolution of sediment source fingerprinting investigations in fluvial systems. Journal of Soils and Sediments, 13, $1658-1675$.

Wang, X. L., Lu, Y. C., \& Wintle, A. G. (2006). Recuperated OSL dating of fine-grained quartz in Chinese loess. Quaternary Geochronology, $1,89-100$.

Weckwerth, P., Przegiętka, K., Chruścińska, A., \& Pisarska-Jamroży, M. (2012). The relation between optical bleaching and sedimentological features of fluvial deposits in the Toruń Basin (Poland). Geological Quarterly, 57(1), 31-44.

Weltje, G. J. (1997). End-member modeling of compositional data: Numerical-statistical algorithms for solving the explicit mixing problem. Mathematical Geology, 29(4), 503-549.

Weltje, G. J., \& von Eynatten, H. (2004). Quantitative provenance analysis of sediments: Review and outlook. Sedimentary Geology, 171(1-4), 1-11.

Wilkinson, M. T., \& Humphreys, G. S. (2005). Exploring pedogenesis via nuclide-based soil production rates and OSL-based bioturbation rates. Soil Research, 43(6), 767-779.

Wilkinson, M. T., Richards, P. J., \& Humphreys, G. S. (2009). Breaking ground: Pedological, geological, and ecological implications of soil bioturbation. Earth-Science Reviews, 97(1-4), 257-272.

Winchell, E. W., Anderson, R. S., Lombardi, E. M., \& Doak, D. F. (2016). Gophers as geomorphic agents in the Colorado Front Range subalpine zone. Geomorphology, 264, 41-51.

Wintle, A. G. (1981). Thermoluminescence dating of late Devensian loesses in southern England. Nature, $289(5797), 479$.

Wintle, A. G. (1993). Luminescence dating of aeolian sands: An overview. Geological Society, London, Special Publications, 72(1), 49-58.

Wintle, A. G., \& Murray, A. S. (1999). Luminescence sensitivity changes in quartz. Radiation Measurements, 30(1), $107-118$.

Wintle, A. G., \& Murray, A. S. (2006). A review of quartz optically stimulated luminescence characteristics and their relevance in singlealiquot regeneration dating protocols. Radiation Measurements, 41(4), 369-391.

Wobus, C., Whipple, K. X., Kirby, E., Snyder, N., Johnson, J., Spyropolou, K., \& Sheehan, D. (2006). Tectonics from topography: Procedures, promise, and pitfalls. Special papers-Geological Society of America, 398, 55-74.

Yanites, B. J., Tucker, G. E., Mueller, K. J., Chen, Y. G., Wilcox, T., Huang, S. Y., \& Shi, K. W. (2010). Incision and channel morphology across active structures along the Peikang River, central Taiwan: Implications for the importance of channel width. Bulletin, 122(7-8), $1192-1208$.

Yawata, T., \& Hashimoto, T. (2004). Identification of the volcanic quartz origins from dune sand using a single-grain RTL measurement. Quaternary Science Reviews, 23(9-10), 1183-1186.

Yukihara, E. G., \& McKeever, S. W. (2011). Optically stimulated luminescence: Fundamentals and applications. Chichester, United Kingdom: John Wiley \& Sons.

Zhang, X. C., Friedrich, J. M., Nearing, M. A., \& Norton, L. D. (2001). Potential use of rare earth oxides as tracers for soil erosion and aggregation studies. Soil Science Society of America Journal, 65(5), 1508-1515.

Zheng, C. X., Zhou, L. P., \& Qin, J. T. (2009). Difference in luminescence sensitivity of coarse-grained quartz from deserts of northern China. Radiation Measurements, 44(5-6), 534-537.

Zular, A., Sawakuchi, A. O., Guedes, C. C., \& Giannini, P. C. (2015). Attaining provenance proxies from OSL and TL sensitivities: Coupling with grain size and heavy minerals data from southern Brazilian coastal sediments. Radiation Measurements, 81, 39-45.

Zular, A., Sawakuchi, A. O., Guedes, C. C., Mendes, V. R., Nascimento, D. R. Jr., Giannini, P. C., \& DeWitt, R. (2013). Late Holocene intensification of colds fronts in southern Brazil as indicated by dune development and provenance changes in the São Francisco do Sul coastal barrier. Marine Geology, 335, 64-77. 